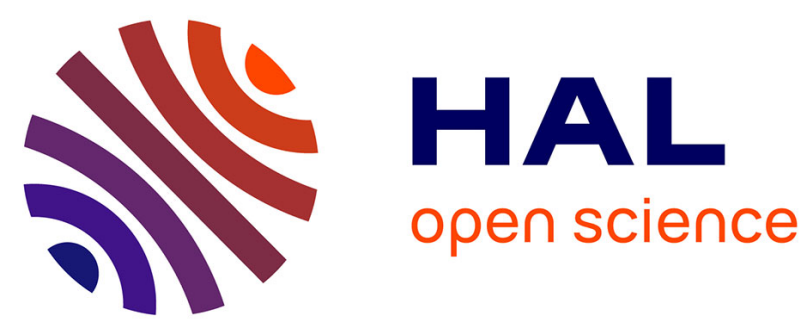

\title{
L'efficacité de la politique des zones d'éducation prioritaire dans les collèges
}

Denis Meuret

\section{To cite this version:}

Denis Meuret. L'efficacité de la politique des zones d'éducation prioritaire dans les collèges. Revue Française de Pédagogie, 1994, 109, pp.41-64. hal-03205810

\section{HAL Id: hal-03205810 \\ https://hal.science/hal-03205810}

Submitted on 4 Jun 2021

HAL is a multi-disciplinary open access archive for the deposit and dissemination of scientific research documents, whether they are published or not. The documents may come from teaching and research institutions in France or abroad, or from public or private research centers.
L'archive ouverte pluridisciplinaire HAL, est destinée au dépôt et à la diffusion de documents scientifiques de niveau recherche, publiés ou non, émanant des établissements d'enseignement et de recherche français ou étrangers, des laboratoires publics ou privés.

\section{(이) $\$$}

Distributed under a Creative Commons Attribution - NonCommercial - NoDerivatives| 4.0 


\section{L'efficacité de la politique des zones d'éducation prioritaire dans les collèges *}

\section{Denis Meuret}

L'article compare les progrès réalisés par les élèves au cours de leurs deux premières années de collège selon qu'ils sont ou non scolarisés en ZEP, en tenant sous contrôle les chances initiales de réussite des élèves.

En français et en mathématiques, les élèves progressent en ZEP un peu moins qu'ils ne progresseraient ailleurs. L'écart est plus grand pour les élèves qui sont initialement en difficulté scolaire ou défavorisés socialement.

Les ZEP semblent avoir un effet positif sur le rapport des élèves à l'école, mais non sur d'autres attitudes ou compétences utiles à leur réussite scolaire ou à leur socialisation.

Ces effets varient d'un collège à l'autre.

D ans les évaluations ou les recherches suscitées par la politique des Zones d'Éducation Prioritaires, une question importante reste sans réponse précise : les élèves qui ont bénéficié de cette politique ont-ils fait davantage de progrès que s'ils n'en avaient pas bénéficié ?

Cette lacune est compréhensible. D'une part, la question elle-même est complexe, car la réussite est une notion dont les dimensions sont nombreuses, car la question de savoir quels élèves précisément sont concernés peut trouver plusieurs réponses. D'autre part, et surtout, il n'est pas facile de disposer des données nécessaires pour tenter d'y répondre.

Une recherche sur les facteurs d'efficacité des collèges, menée à l'initiative du Ministère de
l'Éducation nationale (Direction de l'Évaluation et de la Prospective) de 1990 à 1992, a permis de rassembler certaines de ces données. Celles-ci mesurent en effet les progrès accomplis par les élèves, dans 31 collèges ZEP et 69 hors ZEP, au cours des deux premières années qu'ils passent au collège. De plus, elles portent, non seulement sur deux disciplines fondamentales, le français et les mathématiques, mais aussi sur certaines attitudes des élèves liées tant au processus de leur scolarisation que de leur socialisation.

II a semblé qu'il fallait prendre le risque d'utiliser ces données pour tenter de mesurer les effets de la politiques des ZEP, malgré leurs limites. Elles portent en effet sur le début du collège seulement, sur certaines dimensions de la réussite 
seulement, toutes appréhendées à court terme. Par ailleurs, les 31 collèges étudiés scolarisent une population un peu plus défavorisée que l'ensemble des collèges ZEP.

Un protocole d'évaluation sera construit, à partir de critères suggérés par l'identification des objectifs de la politique ZEP dans les textes qui la fondent, par les évaluations précédentes, et enfin par la situation initiale des élèves de ces collèges ZEP. Ensuite l'efficacité moyenne des collèges ZEP sera étudiée d'abord sur la transmission de compétences en maths et français, puis sur la promotion d'attitudes positives pour la scolarisation ou la socialisation. Enfin, on s'intéressera aux différences entre les collèges ZEP.

\section{LES OBJECTIFS DE LA POLITIQUE DES ZEP SELON LES TEXTES}

Le texte inaugural de la politique des ZEP est la circulaire $n^{\circ}$ 81-238 du ler juillet 1981, intitulée "Zones Prioritaires". Le gouvernement ayant décidé un collectif budgétaire de 11625 emplois pour l'Éducation nationale, ce texte vise à ce qu'ils soient affectés en priorité dans certaines zoneș à la rentrée 1981. II stipule " le but prioritaire [de ces créations d'emploi] est de contribuer à corriger l'inégalité [sociale], par le renforcement sélectif de l'action éducative dans les zones et dans les milieux sociaux où le taux d'échec scolaire est le plus élevé. La politique du gouvernement consiste en effet à subordonner l'augmentation des moyens à leur rendement escompté en termes de démocratisation scolaire ".

Que ce texte mêle "les zones et les milieux sociaux ", tout en proposant une politique de territoire, souligne qu'à travers le territoire, ce sont bien des inégalités sociales qui sont visées. Par la politique des Zones Prioritaires, il s'agit de diminuer l'effet des inégalités sociales sur la réussite scolaire afin d'obtenir qu'un système scolaire plus " démocratique " contribue en retour à diminuer l'inégalité sociale. Le raisonnement qui sous tend la politique des ZEP est le suivant : "l'échec scolaire est plus fréquent dans certaines zones où se concentre une population socialement défavorisée. En renforçant l'éducation éducative dans ces zones, on rapprochera les taux de réussite scolaire des enfants issus de milieux défavorisés de ceux des autres enfants n. Les ZEP visent donc à la fois l'efficacité et l'égalité du système d'enseignement, ou, si l'on préfère, visent davantage d'égalité "par le haut".

Cette circulaire, écrite dans l'urgence de la préparation de la rentrée 1981, fut précisée par celle du 28 décembre ( $N^{\circ} 81-536$; BO spécial $n^{0} 1$ du 21/1/82), qui développe de façon beaucoup plus précise et ample le contenu de la politique des ZEP.

Le premier paragraphe de cette circulaire s'inscrit dans le droit fil de la précédente: [la politique des zones prioritaires] " constitue un instrument privilégié de lutte contre les inégalités devant l'école et, plus généralement, contre les inégalités sociales".

Le second, toutefois, introduit, en filigrane, l'idée que les zones visées ne se contentent pas de concentrer des populations à faibles chances scolaires, mais aggravent les difficultés que ces populations rencontrent: "l'analyse des problèmes que rencontrent dans des aires géographiques précises, les catégories les plus défavorisées met en évidence de fortes relations entre les taux d'échec scolaire et l'environnement socio économique. Autant que l'inadaptation de l'appareil scolaire, c'est la conjonction des difficultés dues aux insuffisances constatées dans différents domaines, et notamment ceux du travail, des loisirs, de l'habitat, de la scolarité, qui caractérise une zone prioritaire".

Or, si la politique ZEP vise moins à réduire les problèmes que l'école pose aux catégories défavorisées, qu'à réduire ceux que ces catégories ont avec l'école "dans des aires géographiques précises ", le raisonnement qui sous tend la politique devient, ou peut devenir, tout autre : "Parmi les inégalités sociales face à l'école, il est probable qu'une partie est issue de comportements individuels qu'une société individualiste ne peut tenir pour entièrement injustes, même si ces comportements sont surdéterminés par des conditions sociales. II est probable aussi qu'une autre partie est issue des conditions de l'enseignement des enfants défavorisés, celles qui proviennent de la ségrégation sociale dans l'habitat, de l'inadaptation des équipements publics à ces catégories ou de leur mauvaise qualité, de la contagion du moindre espoir. Cette part là, issue de facteurs clairement hors d'atteinte de la volonté des jeunes concernés, est à coup sûr injuste et doit être supprimée ». 
Pour aller vite, le premier raisonnement fixe aux ZEP l'ambition d'obtenir que les enfants d'ouvriers y réussissent mieux qu'ailleurs, le second, d'obtenir qu'ils n'y réussissent pas plus mal qu'ailleurs. Pour reprendre les termes de l'analyse que K.R. ROSS fait de la politique australienne d'éducation compensatoire, le premier s'inscrit dans une conception "rédemptrice " de l'éducation, et dans la perspective d'égaliser les progrès moyens de chaque catégorie sociale, le second peut être considéré comme une extension de la conception "libérale " de l'éducation, qui vise à éliminer tous les obstacles externes à l'égalité des conditions de la réussite des élèves.

Une conception "libérale" de type primaire peut considérer qu'elle satisfait à la justice si tout un chacun a accès à des ressources identiques pour son éducation.

En revanche une conception libérale plus élaborée peut considérer que doivent être éradiquées toutes les différences provenant de l'environnement socio-géographique. II ne suffit pas, en effet, d'attribuer des ressources identiques pour assurer l'égalité des chances si, par ailleurs, dans certains endroits, différentes conditions viennent gêner l'usage que les individus peuvent faire de ces ressources. II est parfaitement justifié, dès lors, de tenter de rétablir des conditions environnementales égales par une action compensatoire territoriale.

II faut d'ailleurs noter que ces principes " libéraux" sont congruents avec une politique territoriale, tandis que les premiers considèrent la zone comme l'instrument commode d'une action qui vise en réalité des populations, comme le montre bien la référence quasi permanente, dans les textes qui règlementent les ZEP, à des principes qui relativisent l'idée de zone.

Notons encore que dans cette théorie libérale, les ZEP ne désavantagent personne, ce qui, dans un cadre de justice paretien, est de grand intérêt. Si l'on concentre les moyens dans les ZEP pour que les enfants d'ouvriers y réussissent mieux qu'ailleurs, et si l'on croit vraiment que cette politique va avoir des résultats, on désavantage sciemment les enfants d'ouvriers qui ne sont pas en ZEP (1). En revanche, si la politique ZEP vise à mettre les "pauvres entre eux" au même niveau de chances que les "paurres mêlés aux classes moyennes" on compense un sur-handicap des premiers par rapport aux seconds, de sorte que les seconds ne peuvent plus s'estimer lésés par cette politique.

Ces deux conceptions des ZEP, que nous appellerons ici " sociale " (plutôt que rédemptrice) et " libérale ", vont continuer à être présentes dans les textes réglementaires ultérieurs, mais semble$t$-il, celle qui était au second plan passe au premier.

La circulaire $n^{0}$ 90-028 du 1er février 1990 commence par une référence à l'approche libérale des inégalités (" renforcer l'action éducative dans les zones où les conditions sociales sont telles qu'elles constituent un facteur de risque voire un obstacle pour la réussite scolaire des enfants qui y vivent..." qui se termine logiquement en évoquant la lutte contre l'exclusion plus que l'égalisation des chances ("... et donc, à terme, pour leur insertion sociale $")$.

Que cette approche soit passée au premier plan est sans aucun doute notable. D'autant plus que l'approche sociale subit dans ce texte une inflexion : la lutte contre l'échec scolaire en général s'énonce avant la référence " aux plus défavorisés": "La volonté réaffirmée de promouvoir la réussite de tous les élèves partout et particulièrement dans les zones défavorisées a conduit à relancer cette politique [des ZEP] [...]. L'objectif premier de cette politique est d'obtenir une amélioration significative des résultats scolaires des élèves, (souligné dans le texte, DM), notamment des plus défavorisés".

La circulaire $n^{0} 96-360$ du 7/12/92 synthétise cette conception en une seule phrase simple: "dans les zones difficiles, comme dans le reste du pays, il convient de permettre à tous les élèves de réussir à l'école et d'acquérir une qualification en vue de la meilleure intégration possible "... mais évoque aussi, quoique de façon moins précise que les textes de 1981, l'objectif d'égalité : "les ZEP témoignent du refus de voir se développer dans notre pays une école à plusieurs vitesses et de la volonté d'assurer le plus possible égalité et justice dans le système éducatif $n$.

II n'y aurait pas de sens de reprocher à la politique des ZEP ce type d'ambiguittés : toutes les grandes politiques en présentent du même type, parce que, souvent, ce sont ces ambiguïtés mêmes qui leur permettent d'être voulues, poursuivies, par des forces - sociales, politiques de nature différente. II serait, par ailleurs, erroné de prétendre que cette ambiguilté des objectifs 
interdit l'évaluation de cette politique : tout au plus implique-t-elle que cette évaluation mobilise plusieurs critères.

Une évaluation de la politique ZEP doit donc distinguer trois effets possibles de cette politique. Sur l'échec scolaire, à travers son action sur les élèves les plus en difficultè. Sur l'inégalité sociale devant l'école, à travers son action sur les élèves de catégories sociales défavorisées. Sur l'inégalité territoriale, à travers son action sur le handicap supplémentaire généré par la vie dans certaines zones.

Cette rapide analyse des textes indique aussi les objets ou les biens, si l'on veut, pour lesquels se pose la question de l'égalité : ce qui peut favoriser l'insertion sociale, mais surtout "les résultats scolaires des élèves ".

Ceci est d'ailleurs cohérent avec le fait que les critères retenus pour définir les zones prioritaires ont été d'abord scolaires: les annexes de la circulaire du 28/12/81 présentent la liste de ceux que l'on conseille de retenir, parmi lesquels les critères liés au cursus scolaire des élèves (rapports du nombre d'élèves de $4^{\theta}$ au nombre d'élèves de $5^{\circ}$, du nombre d'élèves de CPPN-CPA au cycle d'observation, etc.) sont les plus nombreux, les plus précis, et ont d'ailleurs été le plus utilisés. C'est aussi cohérent avec le fait que la circulaire de 1992 demande que les évaluations des ZEP portent sur :

1) les résultats des élèves

2) les pratiques pédagogiques mises en œuvre

3) le fonctionnement institutionnel des ZEP.

\section{LES ÉVALUATIONS DES POLITIQUES D'ÉDUCATION COMPENSATOIRE}

L'Angleterre, les USA, l'Australie, les Pays-Bas, entre autres, ont mis en place des actions d'éducation compensatoire à la fin des années soixante. En Angleterre, elles ont fait suite au rapport Plowden (1967), mais sont restées d'une ampleur très limitée (Chrétiennot, in CRESAS, 1985). Elles ont été implantées à une échelle plus large aux USA, dans le cadre de la guerre contre la pauvreté lancée en 1965 à travers des programmes fédéraux, essentiellement " Head start " puis "Follow through ". En 1981, lorsque la politique française fut lancée, ces actions avaient fait l'objet d'évaluations, plutôt négatives.
Ross (1983) écrit: "L'évaluation de "Follow through" suivit 20000 élèves pendant 4 ans pour étudier l'efficacité de 17 modèles d'action compensatoire. Les chercheurs concluent que "aucun des 17 modèles évalués n'a fait la preuve qu'il pouvait compenser de façon significative les conséquences scolaires de la pauvreté" et que l'ensemble du programme Follow through "n'était pas un bon outil pour accroître les scores des enfants de paurres aux tests de connaissances".

Des tentatives néerlandaises, Scheerens (1992) écrit : " Il fut prouvé qu'il n'était pas si simple de redresser la balance [des désavantages sociaux vis-à-vis de l'école] avec des programmes efficaces d'actions compensatoires. En fait, aucun succès éclatant ne peut être établi. II y eut un débat tendu sur la façon dont il fallait interpréter les évaluations disponibles ".

Les ZEP françaises sont donc venues tardivement. Pour des raisons politiques sans doute, mais aussi intellectuelles, dont témoigne le jugement que porte V. Isambert Jamati, en 1973, sur les programmes américains, après en avoir fait une présentation lumineuse. La pédagogie de compensation est tenue par l'auteur pour une " notion paternaliste et réductrice", de sorte que, si ces mouvements sont dignes d'intérêt, ce n'est pas comme possible source d'inspiration, mais comme témoignage sur le système éducatif américain. II semble que l'absence de contenu " critique " de ce type d'actions le condamne, dans le paysage intellectuel français d'alors, à l'indignité politique et intellectuelle $(N$. Isambert Jamati, 1973).

En partie à cause de ce retard, les ZEP françaises présentent par rapport à ces programmes des différences qui peuvent à priori faire espérer de meilleurs effets sur les apprentissages scolaires. D'une part, la culture "critique " avait alors perdu de son influence, de sorte qu'on ne débattait plus de la légitimité qu'avait l'école à transmettre à des enfants pauvres la culture "dominante ", alors que ce débat avait accompagné la mise en œuvre des programmes américains. On a plutôt débattu, en France, de l'intérêt pédagogique d'un détour par l'attention à l'élève, les relations avec ses parents, avec le quartier, pour permettre la réussite scolaire. D'autre part, la définition des ZEP a procédé davantage de critères scolaires quand les zones anglo-saxonnes étaient davantage construites à partir d'indicateurs sociaux portant sur les quartiers. Enfin, il a été 
clair dès le départ que, par eux-mêmes, les moyens supplémentaires ne suffiraient pas à résoudre le problème et qu'il fallait, pour les mériter, présenter des projets spécifiques. On verra à la fin de cette étude que la situation actuelle plaide pour faire un peu moins confiance aux projets et un peu plus aux résultats déjà obtenus, mais, au moins, n'a-t-on encouragé ni la baisse des effectifs par classe ni tout autre usage indifférencié des moyens proposés. De plus, au cours d'assez longues périodes (81-84; 88-92) les ZEP ont bénéficié d'un soutien politique important et d'une certaine mobilisation des services administratifs.

En principe en tous cas, les ZEP françaises ne commençaient pas sans quelques atouts. Parcourir les évaluations déjà proposées de cette politique nous servira d'abord à situer notre étude parmi elles, mais aussi à repérer quelques critères pour notre étude : les membres d'une organisation ont tendance à se comporter de façon à optimiser les critères qui servent à les juger. Les critères utilisés par une évaluation ou les questions que privilégient les chercheurs peuvent donc, à l'instar d'objectifs fixés par une circulaire, servir de guide à l'action, en cohérence ou non avec les dits objectifs.

Que nous apprennent des ZEP les études ou évaluations qu'elles ont suscitées?

On connaît par la DEP certains des moyens que le Ministère de l'Éducation met à leur disposition : trois fois plus de crédits pédagogiques, sept heures d'enseignement supplémentaires pour 100 élèves dans les collèges, des enseignants relativement stables (MENC-DEP, 1992). Remarquons tout de même que les sept heures supplémentaires pour 100 èlèves dans les 800 collèges ZEP représentent moins de $1 \%$ des postes d'enseignants affectés aux collèges.

La pertinence du découpage des ZEP pose problème : elles comprennent des collèges dont la population est relativement peu "défavorisée "; elles sont loin de comprendre l'ensemble des collèges en situation difficile (Trancart, in MENCDEP, 1992)

Les projets des ZEP, les actions menées visent en priorité l'amélioration des apprentissages fondamentaux. "Les priorités dans les écoles et les collèges restent la lecture, l'expression écrite et orale, l'aide au travail des élèves" (LiensolCEuvrard, in MENC-DEP, 1992). Ce constat rejoint l'analyse que V. Isambert Jamati (1989) propose des rapports établis, en 1982, par les coordonnateurs de ZEP à la demande du Ministère. Les actions qui visent à renforcer les apprentissages y sont, également, largement les plus nombreuses, ce dont l'auteur déduit que les accusations de dérive vers "l'animation culturelle" sont infondées.

En revanche, le rapport de l'Inspection générale de l'Administration de l'Éducation nationale, établi à la demande de $M$. JOSPIN en 1988, note que "l'action ZEP a dû faire sa percée dans un espace social fortement quadrillé par des instances étrangères à l'Éducation nationale " et sur un espace politique dominé par les collectivités locales, l'Éducation nationale se retrouvant "d'impulseur, simple partenaire". L'action, de ce fait, " a partout mis l'accent sur l'environnement de l'enseignement plus que sur l'enseignement lui même ".

La forte présence dans les actions ZEP d'un soutien scolaire, d'une aide aux devoirs, souvent peu articulée avec l'enseignement lui même, est compatible avec ces deux jugements a priori différents. D'autre part, l'enquête de Liensol et Cuvrard, qui date de 1990-91, peut témoigner d'un certain recentrage de l'action suite à la circulaire qui a, pour une large part, suivi les recommandations de I'IGAEN.

S'agissant maintenant des effets de cette politique, les jugements, mais aussi les critères auxquels ils se réfèrent, sont divers.

L'effet sur les apprentissages fondamentaux n'est étudié de façon chiffrée que dans Mingat (1983) et dans MENC, DEP (1992).

Mingat a étudié une action ZEP menée dans 9 cours préparatoires et a comparé, toutes choses égales par ailleurs, les progressions de ces élèves avec ceux de sept CP " témoins ". II observe un effet égalisateur de l'action ZEP, en même temps qu'un effet légèrement négatif sur l'efficacité moyenne de l'enseignement.

La DEP a utilisé les évaluations nationales CE2 et $6^{\theta}$ de 1991, et observe qu'appartenir à une ZEP a un effet négatif, toutes choses égales par ailleurs, sur le score observé, effet assez sensible au CE2, très faible en 6ème. La DEP montre aussi que la proportion d'élèves qui quittent le système scolaire depuis le collège (au niveau 6) est plus forte en ZEP qu'hors ZEP, et que l'écart s'est 
creusé, en ce domaine, de 1988 à 1990. Mais les effets de l'appartenance à une ZEP ne sont pas forcément ceux "de la politique ZEP " d'une part et, d'autre part, les élèves qui ont passé leur test en ZEP n'y étaient pas forcément scolarisés auparavant, tandis que tous ceux qui y étaient scolarisés n'y ont pas forcément passé leur test.

Par ailleurs, une appréciation sur les effets de la politique ZEP est portée dans le rapport de l'Inspection générale en 1992. Selon cette appréciation, l'écart de la situation scolaire entre les ZEP et le reste du système scolaire est resté à peu près constant, malgré un accroissement de la précarité et des difficultés dans les ZEP (2).

Selon l'ampleur de la croissance de l'écart social entre les populations en ZEP et hors ZEP, ce jugement signifie que l'efficacité des établissements ZEP, mesurée toutes choses égales par ailleurs, s'est rapprochée sans l'atteindre de l'efficacité des autres, ou a atteint l'efficacité des autres, ou encore l'a dépassée. On verra que les résultats de notre étude ne sont compatibles qu'avec l'hypothèse d'un rapprochement, mais sans l'atteindre, de l'efficacité des autres, et encore, dans leur version la plus optimiste pour la politique ZEP.

Pour présenter les évaluations des autres effets de la politiques ZEP deux distinctions proposées par Donzelot et Estèbe (1993) sont utiles. Selon eux, la politique de développement social urbain, devenue politique de la ville, a opéré une double inflexion, d'un objectif d'intégration à un objectif d'insertion, d'une recherche de la participation à une recherche de l'implication. L'intégration, c'est " l'action conduite selon l'esprit républicain pour assimiler les populations par [entre autres] l'enseignement ". La démarche que recouvre le terme d'insertion "commence là où s'épuise celle conduite au nom de l'intégration ", elle se définirait comme "l'apprentissage du mode d'emploi de la société pour qui se trouve désemparé, exclu"; elle "cherche à augmenter au sein de chaque institution la marge de choix de chaque individu, à la renforcer par des alliances externes qui l'aident à trouver le chemin menant vers son objectif n. L'intérêt de cette distinction est de mettre en lumière que l'Éducation nationale, qui s'est aussi placée dans une logique d'insertion (à travers par exemple les structures du Dispositif d'Insertion des Jeunes par l'Éducation Nationale), a d'abord conçu sa participation à la politique de la ville à travers la politique ZEP, dont la logique vise plutôt l'intégration. II est notable de ce point de vue que le DIJEN ne soit pas évoqué dans la circulaire ZEP du 7 décembre 1992.

II n'est pas sûr que d'insérer dans la politique de la ville une politique dont la logique était autre ait été bénéfique à l'une comme à l'autre. Les dysfonctionnements dont l'Inspection générale de l'Administration se fait l'écho en 1988 pourraient trouver là une partie de leurs causes.

La distinction entre participation et implication, éclaire un autre aspect des évaluations des ZEP. Le modèle participatif " confond ses membres en une seule entité uniforme, spontanée, égalitaire (...) séparée du pouvoir par une barrière constitutive, celle qui distingue démocratie directe et représentation, autogestion et technocratie". L'implication est sollicitée, elle, par des dispositifs c'est-à-dire par a l'assemblage de personnes requises en raison de leurs motivations, mais aussi de leurs compétences (...) avec le souci de les inscrire dans un projet élaboré en commun ". Les textes d'orientation des ZEP, surtout les derniers, se placent plutôt dans la logique de l'implication. Mais de nombreuses évaluations, il est vrai plus anciennes, se placent dans l'autre logique. Les ZEP sont évaluées à l'aune de leur capacité à dépasser, à briser les cloisonnements institutionnels, à favoriser les rencontres de différentes catégories d'éducateurs, à susciter la mobilisation de leurs acteurs (Chambon-Proux, 1988 ; Cresas, 1985), éventuellement pour souligner les obstacles que rencontre une politique ainsi conçue (Plaisance, 1988).

A cette ligne d'évaluation, on peut associer celle qui met en avant les capacités d'innovation des ZEP. Les ZEP sont moins perçues comme le lieu d'une mission propre que comme des laboratoires où doivent s'expérimenter des formules nouvelles d'enseignement. Ce critère est privilégié, bien sûr, par ceux qui voient dans les ZEP la possibilité d'opérer par l'exemple une critique de la "grosse machine Éducation nationale" au nom d'un local inventif et capable de traiter globalement, plutôt que sectoriellement, les problèmes. II est aussi privilégié par l'Administration centrale elle-même (Rapport IGEN 1986, 1992), qui y voit sans doute la possibilité de rendre plus dynamique le réseau des établissements. L'hommage rendu aux ZEP est alors " d'avoir joué un rôle précurseur " (IGEN, 1986). 
Avant de tenter d'évaluer les effets de la politique ZEP, il faut donc avoir à l'esprit qu'elle a été menée par des acteurs pris dans des enjeux divers, des oppositions multiples: Éducation vs enseignement, certes, mais aussi institutionnel vs fusionnel, local vs national, administration vs pouvoirs locaux, et en liaison avec une politique plus vaste - la politique de la ville - dans laquelle son insertion, quelque souhaitable qu'elle ait pu être, n'allait pas de soi.

\section{LA SITUATION DES ÉLEVES À L'ENTRÉE DES COLLĖGES ZEP}

L'évaluation réalisée par la Direction de l'Évaluation et de la Prospective (MENC-DEP,1992) donne deux indications essentielles pour caractériser la population scolarisée dans les collèges ZEP.

La proportion d'élèves étrangers est l'un des facteurs les plus discriminants des ZEP par rapport aux autres établissements : dans le premier cycle du second degré, ils sont $9,5 \%$ dans l'ensemble des collèges publics et $21,7 \%$ en ZEP, soit 2,3 fois plus.

Les élèves de 6ème des collèges ZEP sont aussi, plus souvent qu'ailleurs, en retard : $17,8 \%$ d'entre eux présentent des retards de 2 ans ou plus, contre $11,3 \%$ dans l'ensemble des collèges.

Les données recueillies ici permettent de compléter ce portrait, en rappelant cependant que les

Tableau 1 : Les élèves de $6^{\circ}$, en ZEP et hors ZEP, dans les 100 collèges de l'échantillon

\begin{tabular}{|c|c|c|c|c|c|}
\hline & \multicolumn{3}{|c|}{ Valeurs brutes } & \multicolumn{2}{|c|}{ Valeurs réduites } \\
\hline & $\begin{array}{c}\text { ZEP } \\
N=1782\end{array}$ & $\begin{array}{l}\text { Hors ZEP } \\
\mathrm{N}=3770\end{array}$ & $\begin{array}{l}\text { Signif. } \\
\text { écart }\end{array}$ & ZEP & Hors ZEP \\
\hline \multicolumn{6}{|l|}{ Caractéristiques générales } \\
\hline Age $\ldots \ldots \ldots \ldots \ldots \ldots$ & 11,65 & 11,39 & S & & \\
\hline $\begin{array}{l}\text { Catégorie professionnelle } \\
\text { et sociale }(1) \ldots \ldots \ldots \ldots \ldots\end{array}$ & 0,39 & 0,18 & S & & \\
\hline Proportion d'élèves étrangers $\ldots \ldots$ & 0,21 & 0,05 & S & & \\
\hline \multicolumn{6}{|l|}{ Connaissances } \\
\hline Maths $\ldots \ldots \ldots \ldots \ldots \ldots \ldots \ldots$ & 0,64 & 0,72 & S & $-0,32$ & 0,14 \\
\hline Français $\ldots \ldots \ldots \ldots \ldots \ldots \ldots$ & 0,45 & 0,54 & S & $-0,33$ & 0,14 \\
\hline \multicolumn{6}{|l|}{$\begin{array}{l}\text { Capacités requises par l'univers } \\
\text { scolaire (2) }\end{array}$} \\
\hline ORGanisation $\ldots \ldots \ldots \ldots \ldots$ & 0,69 & 0,92 & S & $-0,13$ & 0,05 \\
\hline MOTivation $\ldots \ldots \ldots \ldots \ldots$ & 0,86 & 0,81 & $\mathrm{~S}$ & 0,06 & $-0,03$ \\
\hline Image SCOLaire $\ldots \ldots \ldots \ldots \ldots$ & 0,21 & 0,25 & NS & 0,00 & 0,00 \\
\hline COOPération $\ldots \ldots \ldots \ldots \ldots \ldots$ & 0,51 & 0,62 & $\mathrm{~s}$ & $-0,10$ & 0,05 \\
\hline DEMObilisation $\ldots \ldots \ldots \ldots \ldots$. & $-1,04$ & $-1,01$ & NS & 0,04 & $-0,01$ \\
\hline \multicolumn{6}{|l|}{$\begin{array}{l}\text { Capacités requises } \\
\text { par la socialisation (2) }\end{array}$} \\
\hline CIVisme $\ldots \ldots \ldots \ldots \ldots \ldots$ & 11,80 & 13,80 & $\mathrm{~S}$ & $-0,21$ & 0,09 \\
\hline Image SOCiale $\ldots \ldots \ldots \ldots \ldots$ & 0,56 & 0,58 & S & $-0,06$ & 0,03 \\
\hline SOCIABilité $\ldots \ldots \ldots \ldots \ldots \ldots$ & 0,17 & 0,22 & $\mathrm{~S}$ & $-0,15$ & 0,07 \\
\hline AGRessivité $\ldots \ldots \ldots \ldots \ldots \ldots$ & 6,29 & 5,68 & NS & 0,11 & $-0,05$ \\
\hline
\end{tabular}

(1) Proportion d’êlèves appartenant à une CSP défavorisée, soit: ouvriers non qualifiés, ouvriers agricoles, retraités autrefois employés ou ouvriers, chómeurs n'ayant jamais travaillé, inactifs. Cette définition des catégories défavorisées, assez restrictive, sera utilisée pour toute cette étude.

(2) Voir la définition de ces capacités dans GRISAY (1993).

Lecture des valeurs réduites : En mathématiques (MATHS), le score moyen des élèves de ZEP est inférieur, au score moyen de l'ensemble des éléves, de 0,32 fois l'écart-type de 1 distribution de ce score parmi l'ensemble des éléves. La " réduction * permet de comparer l'écart ZEP/non ZEP de deux variables en neutralisant l'effet sur cet écart de la plus ou moins grande ampleur de chaque distribution.

Lecture des valeurs brutes des scores d'attitudes: Pour tous les scores d'attitude, sauf CIV et AGR, l'échelle utilisée varie de -2 à +2 . Un score de -2 correspond à une réponse tout à fait négative à tous les items de l'échelle. Un score de +2 correspond à une réponse positive (* tout à fait d'accord *) à tous les items de l'échelle. CN est noté sur 24 : la valeur du score est le nombre de réponses a démocratiques n aux items. 
élèves de notre échantillon de collèges ZEP sont dans une situation un peu plus difficile que la moyenne des collèges ZEP (cf. annexe 1).

Les élèves de ZEP se distinguent surtout des autres, à leur entrée au collège, par la nationalité, la catégorie sociale. Leur handicap socio-scolaire se double d'un déficit par rapport à certaines attitudes désirables dans l'univers scolaire ou pour leur socialisation. Mais ce déficit est léger et, de plus, il n'est pas général, même sur le petit nombre de dimensions prises en compte. En particulier, parmi les capacités que requiert l'univers scolaire, ils présentent des lacunes en matière d'organisation de leur travail (ORG) et de propension à la coopération (COOP), mais ils ne se déclarent pas plus enclins que les autres au découragement devant les difficultés (DEMO), ils ne se sentent pas hors des normes de l'école (SCO) et ils se déclarent même plus motivés que les autres (MOT). On sait que les enfants de catégories défavorisées sont, plus que d'autres, enclins à émettre des réponses désirables. Mais il n'est pas obligatoire de l'interpréter comme le travestissement d'une vérité cachée moins positive. Cela fait partie de l'attitude de l'individu, et peut être mobilisé par l'école au même titre qu'une attitude plus authentiquement - pour autant que ce mot ait un sens ici - positive. Ce qu'on peut observer, donc, c'est que les élèves de ZEP ne déclarent pas, au début de la $6^{\circ}$, un plus mauvais rapport à l'école que les autres, mais qu'ils manquent d'outils ou de dispositions (méthodes d'organisation, propension à la coopération) que l'école devrait pouvoir leur procurer.

Leurs capacités sociales sont un peu plus faibles que celles de leurs camarades hors ZEP : Ils sont moins sociables et leurs attitudes civiques sont moins bonnes, ils sont (verbalement) un peu plus agressifs que les autres. Cependant, ils se sentent (presqu') autant que les autres capables de se faire aimer et apprécier (SOC). Leur problème est donc moins, semble-t-il, d'être isolés, rejetés que de maîtriser moins bien les comportements qui facilitent la vie collective.

Ce portrait des élèves de ZEP appelle deux remarques.

1) La grande majorité des collèges étudiés sont en ZEP depuis plus de 5 ans. La plupart de ces enfants ont dû fréquenter des écoles primaires de ZEP. Mais nous ne savons pas en quelle proportion exactement. D'autre part, les ZEP primaires peuvent avoir réduit les handicaps de leurs élèves, sans les supprimer complètement. II est donc impossible de tirer, de cette analyse de la situation au début de la sixième, une évaluation de l'efficacité des ZEP pour l'enseignement primaire.

2) Les textes juridiques mettent l'accent sur les enjeux scolaires des ZEP et sur les critères scolaires pour décider de l'attribution du label, et ceci bien davantage que dans les expériences étrangères d'éducation compensatoire. Pourtant, il semble, d'après une analyse de régression dite discriminante, que la coupure entre les colleges ZEP et les autres soit plus nette sur des critères sociaux que sur des critères de niveau scolaire. Les caractéristiques sociales des élèves (CSP, Nationalité) prédisent davantage que leurs caractéristiques scolaires (Age, Niveaux en français et en mathématiques) leur appartenance à une ZEP (tableau 2).

Pour une part, cela peut venir de ce que les critères initialement retenus pour choisir les ZEP portaient sur la trajectoire dans le système scolaire (retards, orientations) et non sur le niveau des connaissances dont l'évaluation systématique

Tableau 2. - Caractéristiques permettant de discriminer les populations ZEP et NZEP

\begin{tabular}{|c|c|c|c|}
\hline Variables & R2 & $\begin{array}{c}F \text { (capacité prédictive } \\
\text { de la variable) }\end{array}$ & Significativité \\
\hline EL - Étranger & 0,078 & 548 & S \\
\hline CSP - Défavorisée & 0,069 & 477 & $S$ \\
\hline Score Français $6^{\mathrm{e}}$ & 0,063 & 438 & $S$ \\
\hline Score Maths $6^{e}$ & 0,063 & 434 & $\mathrm{~S}$ \\
\hline Année de naissance & 0,020 & 130 & $S$ \\
\hline
\end{tabular}


n'existait pas au début de l'opération. Or, on sait que les trajectoires scolaires sont davantage marquées socialement que l'acquisition des connaissances (DURU-BELLAT et MINGAT, 1993). Mais il est possible aussi que la logique même d'une action territoriale, conduise à privilégier des critères de nature sociale plus que purement scolaire.

Quoi qu'il en soit, cette situation rappelle l'ambiguiité relevée lors de l'analyse des évaluations précédentes, entre les objectifs scolaires et sociaux de la politique ZEP. Cette ambiguïté peut être dénoncée, comme la preuve que derrière la volonté de favoriser l'égalité sociale par une politique scolaire, se "cache " une politique qui manifeste surtout que l'école s'efforce, par des moyens accrus, de prendre en charge, pour la neutraliser, une population "à risques " On peut aussi trouver qu'un tel effort n'a rien de honteux, fait au contraire partie des missions premières de l'école. En ce cas, on est quant même conduit à se demander si la politique ZEP était la mieux adaptée à cet effort ou si, inversement, une politique aux objectifs aussi fortement scolaires n'aurait pas du s'appliquer à une population davantage discriminée sur des critères scolaires.

En tout état de cause, il importe d'autant plus de savoir si la politique des ZEP a effectivement contribué à réduire l'écart de civilité (appelons ainsi ce qui aide à vivre ensemble dans une cité) entre les jeunes, que le découpage mème des ZEP semble indiquer une forte préoccupation à cet égard.

\section{UN CANEVAS POUR L'ÉVALUATION DES ZEP}

Proposer une évaluation des ZEP suppose qu'on définisse les domaines sur lesquels on va tester leur effet mais aussi l'enjeu que l'on fixe aux établissements ZEP pour décider si leurs résultats signifient plutôt la réussite ou l'échec de cette politique.

\section{1) Les domaines}

On peut évidemment s'intéresser aux taux d'orientation, de redoublement, de poursuites d'études que l'on peut observer dans les ZEP. II est cependant clair qu'on saisit à travers de tels indicateurs l'attitude du système scolaire vis-à-vis des élèves de ces zones, ce qui n'est pas sans intérêt mais ne renseigne pas sur l'efficacité réelle de ces établissements vis-à-vis de leurs élèves. Celle-ci ne peut se mesurer qu'aux évolutions de connaissances, de compétences et/ou d'attitudes que l'on peut observer chez ces élèves, ou au niveau qu'elles ont atteint. Sans doute, une étude comme celle-ci ne peut-elle que mesurer les effets immédiats de la fréquentation des collèges ZEP. Mais, outre que rien n'interdit d'interpréter les effets immédiats dans la perspective de leurs conséquences futures, il est à craindre qu'une baisse progressive de l'influence reçue ne soit plus probable que l'éclosion tardive d'effets bénéfiques d'abord invisibles (3).

Les analyses qui précèdent justifient de plusieurs façons de ne pas évaluer seulement les acquis dans les principales disciplines, mais aussi l'évolution des attitudes ou comportements des élèves. Les textes réglementaires eux-mêmes les évoquent, bien que sur le mode mineur. Les évaluations (IGEN 86, en particulier) mettent parfois en avant les progrès obtenus en matière de socialisation, de rapport à l'école, d'épanouissement individuel mais, faute d'avoir pu mesurer ces progrès, elles ont pu être soupçonnées de n'en faire état que pour compenser l'absence de progrès mesurables dans le domaine cognitif. Ensuite, les ZEP ont été découpées comme si des critères sociaux, et à travers eux, comportementaux avaient eu quelqu'importance à côté des critères purement scolaires. Enfin, les évaluations révèlent que les collèges ZEP ont fonctionné avec de nombreux partenaires (animateurs d'activités périscolaires, pouvoirs locaux soucieux de prévenir la délinquance, chefs de projets DSQ, ...) intéressés à ces dimensions et prêts à en rappeler l'importance.

\section{2) Les enjeux}

Comment décider, dans le cas des apprentissages fondamentaux comme dans celui des attitudes des élèves, si les ZEP ont réussi ou échoué ?

Selon l'opinion la plus partagée, le succès des ZEP se jugerait à ce qu'elles auraient supprimé, ou diminué l'écart qui sépare leurs établissements des autres. Dire (IGEN, 1992) que l'écart est resté constant dans la réussite observable des élèves tandis qu'il s'est creusé entre les caractéristiques des élèves pris en charge par les ZEP et les autres s'inscrit dans cette approche. 
Le jugement de l'Inspection générale rappelle cependant que la mesure du niveau brut doit se combiner avec la prise en compte de la nature des élèves scolarisés en et hors ZEP. On doit introduire dans l'analyse la notion d'efficacité des établissements ZEP, c'est-à-dire leur capacité à faire progresser leurs élèves davantage que leurs caractéristiques initiales ne le laissaient prévoir.

Dans l'optique sociale, l'objectif est que le handicap social ou scolaire soit moins prégnant dans les ZEP qu'ailleurs, autrement dit que les collèges ZEP soient plus efficaces que les autres. En poussant cette logique à son terme, on pourrait même préciser : pour les élèves faibles ou appartenant à une catégorie sociale défavorisée, l'efficacité moyenne du collège n'important que secondairement.

Dans l'optique libérale tous les élèves scolarisés en ZEP subissent un préjudice que la politique ZEP doit compenser. Pour que la politique ZEP ait réussi, il faut et il suffit dès lors que chaque élève de ces zones progresse en ZEP de la même façon qu'il progresserait ailleurs. Cela signifie que non seulement l'élève moyen des ZEP doit progresser autant qu'il le ferait ailleurs, mais encore que chaque sous-catégorie (en particulier les élèves défavorisés socialement ou/et en difficulté scolaire à l'entrée au collège) doit faire de même.

Comparer l'évolution sur deux ans d'élèves en et hors ZEP permet donc de répondre sensiblement aux mêmes questions qu'une comparaison de l'écart entre les collèges ZEP et les autres avant et après la mise en œuvre de cette politique, pourvu que l'on corrige les progrès observés chez les élèves, en tenant compte des chances de progresser que leur conférait leur situation (niveau scolaire, âge, catégorie sociale...) au moment d'entrer dans le collège.
On verra cependant plus loin que, dans le cadre de l'optique libérale, l'absence de situation de référence pose quand même une limite à l'évaluation.

Un dernier élément doit être apporté au dossier avant l'évaluation proprement dite : il ne convient pas d'attendre de la politique ZEP des effets miraculeux. D'une part les évaluations étrangères d'actions compensatoires ont montré des effets de faible ampleur. D'autre part, même si tous les collèges ZEP devenaient très efficaces, l'effet pour chaque élève serait limité, le poids des déterminants individuels restant fort en tout état de cause. Inversement, un collège qui améliore, même faiblement, le sort de tous les élèves qui le fréquentent, finit par améliorer le sort de beaucoup de monde...

\section{L'EFFET DES ZEP SUR LES PROGRÈS EN MATHÉMATIQUES ET EN FRANÇAIS}

Les scores des élèves de ZEP sont inférieurs aux scores des autres élèves, en français et en mathématiques. Deux ans après leur entrée au collège, l'écart s'est creusé, dans les deux disciplines, au détriment des élèves scolarisés en ZEP (tableau 3).

II est difficile de tirer des conclusions de ce constat. On sait en effet que les écarts se creusent entre les élèves au cours du cycle d'observation, au détriment des élèves socialement défavorisés et/ou en difficulté scolaire. D'autre part, certains élèves ont pu quitter les collèges de l'échantillon entre les deux épreuves.

Nous avons donc eu recours à un modèle de régression multiple, qui permet d'évaluer l'effet de

Tableau 3. - Scores bruts aux épreuves de mathématiques et de français

\begin{tabular}{|c|c|c|c|c|c|c|c|c|}
\hline & \multicolumn{4}{|c|}{ Début sixième } & \multicolumn{4}{|c|}{ Deux ans plus tard } \\
\hline & $\mathrm{n}$ & ZEP & NZEP & $\frac{\text { ZEP }}{\text { NZEP }}$ & $\mathrm{n}$ & ZEP & NZEP & $\frac{\text { ZEP }}{\text { NZEP }}$ \\
\hline Français $\ldots \ldots \ldots \ldots \ldots \ldots$ & 7412 & $45,3 \%$ & $53,9 \%$ & 0,84 & 7010 & 42,9 & 54,0 & 0,79 \\
\hline Mathématiques $\ldots \ldots \ldots \ldots \ldots$ & 7443 & $64,2 \%$ & $72,3 \%$ & 0,89 & 7097 & 51,2 & 63,4 & 0,81 \\
\hline
\end{tabular}


Tableau 4. - Impact des différentes caractéristiques des élèves sur leurs progrès en maths et français

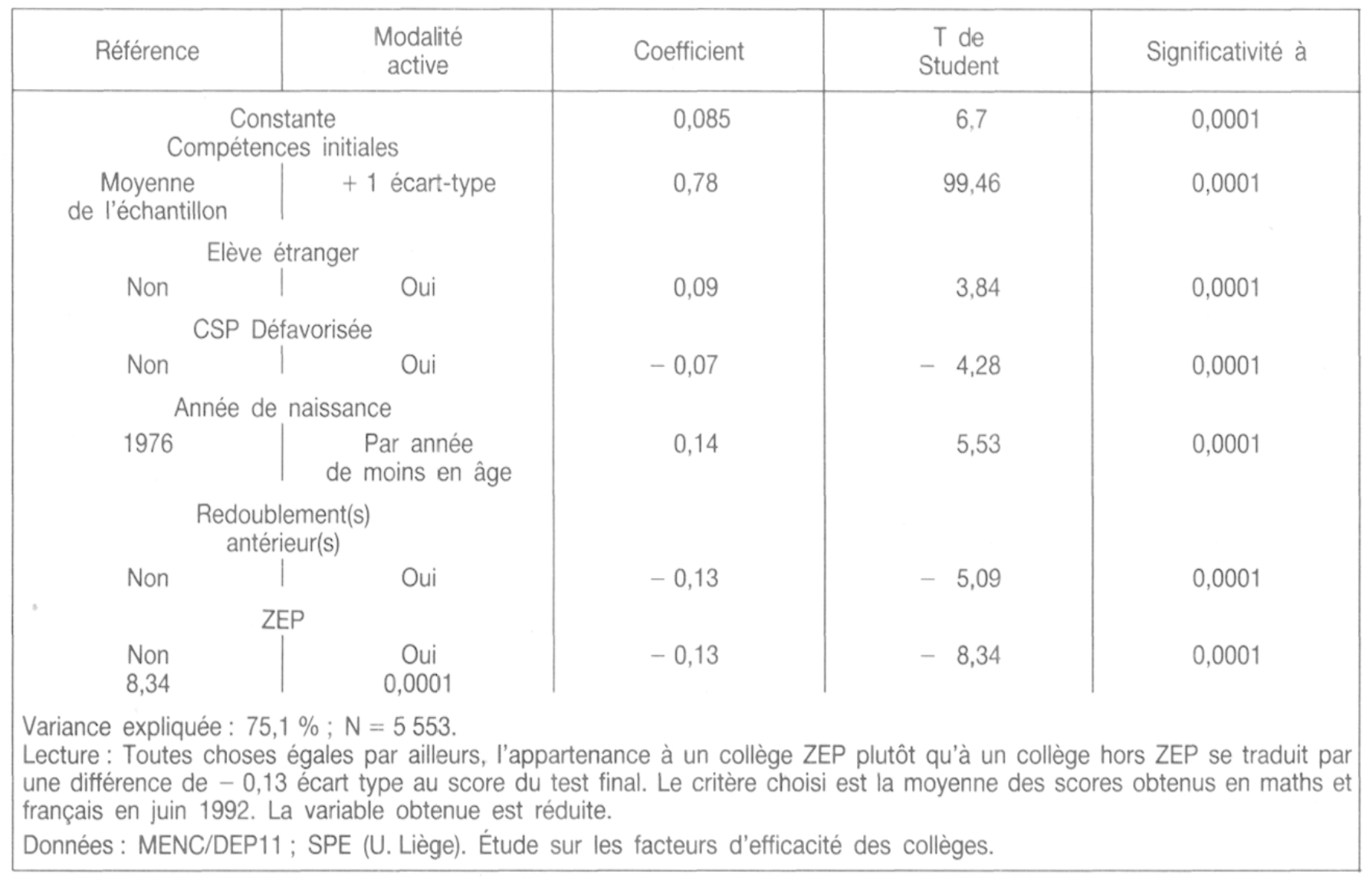

l'appartenance aux collèges ZEP sur les scores obtenus en fin de $5^{\mathrm{e}}$ en maths et en français, en neutralisant l'effet des caractéristiques initiales des populations fréquentant les deux types de collèges : Age, Nationalité, appartenance ou non à une catégorie défavorisée, scores obtenus en français et maths en début 6ème (cf. Tableau 4)

Comme on pouvait s'y attendre, le déterminant le plus important des compétences mesurées par le score final est, de loin, le niveau initial de ces compétences. A compétences initiales égales, les autres caractéristiques de l'élève (Age, milieu social, nationalité, passé scolaire) n'ont qu'une incidence marginale, bien que statistiquement significative. On peut quand même noter que cette étude confirme que, toutes choses égales par ailleurs, la nationalité étrangère n'est pas un handicap.

La dernière ligne du tableau 4 donne une première réponse à la question de "l'effet ZEP": toutes choses égales par ailleurs (quant à l'âge, aux compétences initiales, au milieu social, à la nationalité et au passé scolaire de l'élève), la fréquentation pendant deux ans d'un collège ZEP se traduit en moyenne par un score final (moyenne français et maths) inférieur d'environ un dixième d'écart type à celui qu'auraient obtenu hors ZEP des élèves présentant les mêmes caractéristiques initiales.

Cette estimation de "l'effet ZEP " mérite cependant d'être acceptée avec quelque réserve. La technique utilisée, l'analyse de régression, présente en effet un certain risque de biais lorsqu'elle est utilisée pour tenir sous contrôle les caractéristiques de groupes d'élèves très différents entre eux, comme c'est le cas ici (4). Comme le montre Heuchenne (1993), ce biais a pour conséquence un risque de sous estimation de l'éventuel effet bénéfique du "traitement " d'un groupe d'individus, lorsque l'appartenance au groupe "traité " (ici, par la politique ZEP) est corrélé négativement avec le score obtenu au test initial qui est utilisé comme variable de contrôle. C'est le cas ici, puisque les élèves de ZEP sont plus faibles, en moyenne, à leur entrée en 6ème, que les autres.

Nous avons donc procédé, à titre de vérification, à une analyse complémentaire au moyen d'une analyse de structure de covariance (LIS- 
REL), qui permet d'obtenir une estimation de l'effet ZEP non entachée de cet artefact. L'annexe 2 présente cette analyse.

Ses résultats confirment ceux de la régression précédente, en les atténuant: l'appartenance à une ZEP influence, faiblement mais négativement, les progrès réalisés par les élèves en maths et en français au cours de leurs deux premières années de collège. L'estimation de l'effet négatif de l'appartenance à une ZEP est ramené cependant de 0,130 écart type à 0,042 écart type. Cet effet reste significatif statistiquement.

La politique ZEP a donc échoué à créer une situation où, en moyenne, les élèves scolarisés dans ces zones réussiraient mieux que leurs caractéristiques ne le laissent espérer, du moins au cours des deux premières années du collège. En réalité, ils réussissent même un peu moins bien qu'ils ne réussiraient ailleurs.

Dans l'approche sociale, on ne prend pas en considération le fait qu'il pourrait exister au départ, un sur-handicap de nature socio-territoriale. Celui qu'on observe aurait donc été créé par la politique ZEP.

Toutefois, il est possible que cet effet moyen soit le résultat d'effets contradictoires : une partie de la population en ZEP faisant mieux que prévu, une autre faisant beaucoup moins bien que prévu. Or, si, par exemple, ceux qui font mieux que prévu sont les élèves socialement défavorisés ou scolairement faibles, la politique des ZEP aura néanmoins réussi à diminuer l'écart entre ces catégories et les autres.

Dans cette approche, il importe donc de comparer l'effet des caractéristiques les plus importantes des élèves, selon qu'ils sont en ZEP ou non, sur les progrès qu'ils ont réalisé en maths et en français. Si, en ZEP, l'effet négatif de l'appartenance à un milieu social défavorisé est plus faible qu'hors des ZEP, cela signifiera que les élèves défavorisés, toutes choses égales par ailleurs, réussissent mieux en ZEP qu'hors ZEP.

Cette comparaison est résumée dans le tableau 5 , obtenu en conduisant deux analyses de régression multiple distinctes, l'une sur les élèves ZEP, l'autre sur les élèves hors ZEP.

La ligne " constante " du tableau 5 redonne le résultat obtenu précédemment, savoir que l'effet moyen de l'appartenance à un collège ZEP est
Tableau 5. - Infiuence des compétences initiales, de la nationalité et du millou social en et hors ZEP

\begin{tabular}{|c|c|c|c|}
\hline & Ensemble & $\begin{array}{l}\text { Elèves } \\
\text { en ZEP }\end{array}$ & $\begin{array}{c}\text { Elèves } \\
\text { hors ZEP }\end{array}$ \\
\hline & 0,09 & $\begin{array}{c}\text { Constante } \\
-0,06\end{array}$ & $+0,09$ \\
\hline & \multicolumn{3}{|c|}{ Coefficients de régression } \\
\hline $\begin{array}{l}\text { Compétences } \\
\text { initiales ...... }\end{array}$ & 0,78 & 0,79 & 0,77 \\
\hline $\begin{array}{l}\text { Elève } \\
\text { étranger ...... }\end{array}$ & 0,09 & 0,09 & 0,11 \\
\hline Milieu social . . & $-0,18$ & $-0,10$ & $-0,05$ \\
\hline$N \ldots \ldots \ldots$ & 5553 & 1782 & 3770 \\
\hline
\end{tabular}

négatif sur les progrès réalisés en français et en maths.

Les effets différentiels des ZEP se lisent à travers les coefficients de régression. Ils sont d'ampleur faible mais leur sens est l'inverse de ce qu'on pouvait espérer : le fait d'avoir de bonnes compétences initiales est, évidemment, bénéfique (le coefficient de régression est positif), mais l'est un peu plus en ZEP que hors ZEP, ce qui veut dire que l'écart se creuse un peu plus entre les bons élèves et les autres, en ZEP qu'hors ZEP.

$\mathrm{Si}$, d'ailleurs, on répartit les élèves en trois catégories selon leur pronostic scolaire (à partir de leur âge, de leurs compétences initiales et de l'existence de redoublements antérieurs), leurs progrès se répartissent ainsi: le progrès des élèves dont le pronostic scolaire est défavorable ou moyen est significativement inférieur en ZEP à ce qu'il est hors ZEP, en maths comme en français. En revanche, les progrès des élèves dont le pronostic scolaire est favorable diffèrent peu selon qu'ils sont en ZEP ou non. L'écart entre eux est en deçà de la différence significative minimale, il est même plutôt favorable aux ZEP pour les progrès en maths. Autrement dit, à l'inverse de ce que croient beaucoup de parents, être scolarisé en ZEP n'a pas d'inconvénient pour un bon élève, au moins pour l'apprentissage des disciplines fondamentales.

Etre étranger est, toutes choses égales par ailleurs, corrélé positivement aux acquisitions en français et en maths, mais cet " avantage " relatif que l'on retire du fait d'être étranger est un peu plus faible en ZEP qu'ailleurs.

Appartenir à un milieu social défavorisé est un handicap (le coefficient de régression est néga- 
tif)... et cet handicap est un peu plus fort en ZEP qu'ailleurs (5). En ZEP, l'écart se creuse davantage qu'ailleurs entre les enfants d'ouvriers non qualifiés et les autres.

La considération des effets différentiels de l'appartenance aux ZEP aggrave donc plutôt le diagnostic : nous risquons de devoir conclure que la politique ZEP aggrave (légèrement) des inégalités qu'elle était censée réduire. Nous observons, quoiqu'il en soit, que l'appartenance à une ZEP aggrave les inégalités, qu'elles soient sociales ou scolaires, entre les élèves.

Dans l'approche libérale, on suppose qu'existait au moment de la création des ZEP un effet contextuel négatif dans les zones. Les résultats observés permettent donc de dire que, en moyenne, les collèges ZEP n'ont pas réussi à supprimer cet effet contextuel, et que donc les objectifs de la politique n'ont pas été atteints. En revanche, ils ne permettent pas de dire si la politique ZEP a accru, diminué ou laissé intact cet effet. Ils ne permettent pas non plus de dire si cette politique est en train de le réduire ou de l'accroître.

A. Grisay (1993) observe, en 1990-1992, un effet contextuel sous la forme suivante : à niveau initial en français et en maths égal, la progression moyenne est plus faible dans les collèges fréquentés par une plus forte proportion d'élèves défavorisés. II est probable que c'est cet effet contextuel que l'on observe par la différence des progressions en et hors ZEP.

Mais son existence est compatible aussi bien avec l'optique "sociale", et l'idée que la politique ZEP aurait créé cet effet, qu'avec, dans l'optique "libérale ", l'idée qu'elle l'aurait réduit. On est donc ramené à l'interrogation précédente, ayant seulement constaté par une autre voie que la politique ZEP n'a pas réussi à faire disparaître l'effet contextuel des collèges français.

Une comparaison du fonctionnement des collèges ZEP et hors ZEP éclaire ces résultats. Les caractéristiques des collèges efficaces - i.e. qui font progresser leurs élèves davantage qu'attendu d'après leur niveau initial - sont rarement plus fréquentes en ZEP qu'hors ZEP, beaucoup y sont significativement moins fréquentes (Grisay, 93).

Sans doute, les collèges ZEP différent entre eux. Ce qui est vrai de leur ensemble n'est pas vrai de chacun. Mais ce qu'on observe en moyenne ne laisse pas présager une grande efficacité.

D'abord les collèges ZEP n'arrivent pas à endiguer la plus grande indiscipline de leur public. Cela se répercute sur le temps consacré au travail en classe. Or, discipline et durée du travail sont, ensemble, la variable la plus fortement corrélée à l'efficacité. Ensuite les pratiques pédagogiques sont plus souvent hétérogènes dans les collèges ZEP. Les enseignants ont moins souvent des relations chaleureuses avec leurs élèves. D'après les déclarations des élèves, on étudie en ZEP une moins grande partie du programme qu'hors ZEP.

Au niveau de l'établissement, on observe, outre l'indiscipline, que les règles de fonctionnement paraissent moins claires dans les collèges ZEP, que les élèves y ont moins de droits et de responsabilités, alors que ces variables sont associées à l'efficacité.

Enfin, les administrations - régionale et d'État - ont quelque responsabilité dans le fait que la taille des collèges ZEP est plus importante que celle des autres. En 1991-1992, la taille moyenne des collèges publics était de 516 élèves, contre 538 pour les collèges ZEP. Dans le même sens, et de façon peut être plus significative, la taille des collèges dont la situation sociale et.scolaire a été repérée comme la plus difficile - collèges dits " sensibles" - était de 671, contre 517 pour l'ensemble des collèges publics (Trancart, 1992). Or, s'il semble que les écarts de connaissances entre élèves initialement forts et faibles se creusent davantage dans les petits collèges, on observe aussi que l'efficacité moyenne est plus forte dans les petits collèges, pour la progression des connaissances. La progression des attitudes propices à la scolarisation progresse, elle, davantage dans les petits collèges, lorsque la proportion d'élèves étrangers y est forte ce qui est, bien sûr, souvent le cas en ZEP.

Au total, il n'est pas étonnant que les collégiens de ZEP trouvent la qualité de leur vie à l'école moins bonne, sentiment lui même associé aux collèges les moins efficaces. La réputation des collèges ZEP est d'ailleurs mauvaise, selon les enseignants qui y travaillent, et aussi selon les usagers: l'arrivée d'élèves au cours du cycle d'observation y est moins fréquente que dans les collèges hors ZEP, et le niveau scolaire 'des arrivants est nettement plus bas que celui des élèves 
qui intègrent dans les mêmes conditions les collèges hors ZEP.

Deux aspects seulement du fonctionnement des collèges ZEP semblent adaptés à leur public et porter la trace de cette politique : les enseignants connaissent mieux qu'hors ZEP les parents de leurs élèves. On y pratique davantage le soutien scolaire qui semble bien être un facteur d'efficacité, en particulier lorsqu'il est pratiqué sur des élèves " défavorisés".

La première conclusion de cette étude est que, si les écarts de connaissances sont nets entre élèves de ZEP et hors ZEP, les écarts de performances corrigées entre les collèges ZEP et hors ZEP sont plutôt faibles. Compte tenu du niveau initial et des caractéristiques sociales des élèves de ZEP, les progrès qu'ils font ne sont pas très éloignés de ceux qu'ils feraient ailleurs. Ces écarts sont, donc, plutôt faibles, mais, c'est la seconde conclusion, plutôt négatifs, au détriment des élèves de ZEP.

Les élèves de ZEP progressent un peu moins que ce que l'on attendrait au vu de leurs caractéristiques sociales et scolaires initiales, ce qui signifie qu'ils progressent moins que les élèves hors ZEP qui ont les mêmes caractéristiques qu'eux. C'est vrai en moyenne, c'est encore plus vrai pour les enfants de catégories sociales défavorisées, pour les étrangers, c'est vrai pour tous sauf pour les bons élèves...

Si donc on suppose qu'il n'y a pas de handicap particulier à être scolarisé dans une zone sensible, si les ZEP sont simplement une façon commode d'atteindre une population à faibles chances scolaires, il faut considérer comme probable que la politique ZEP a échoué à diminuer l'inégalité devant l'école, comme d'ailleurs l'échec scolaire moyen - et nón seulement qu'elle a échoué, mais qu'elle a plutôt légèrement aggravé les choses.

Si l'enjeu véritable des ZEP est d'annuler le surhandicap que le contexte scolaire et social des collèges des ZEP génère, il est sûr que, dans ces collèges, la politique ZEP n'a pas réussi à combler ce handicap, mais on ne peut exclure qu'elle ne l'ait pas aggravé ou qu'elle l'ait réduit.

Pour évaluer la politique ZEP, savoir s'il existait avant cette politique un effet contextuel négatif dahs ces zones est donc important. Malheureusement, les résultats disponibles ne donnent pas de réponse sûre à cette question, du moins à notre connaissance. Le rapport Coleman (cf. Cherkaoui,1979) et Bloom (1986, à propos de l'influence du groupe de pair), établissent l'existence d'un tel effet aux États Unis. Wilms (1985) l'observe au royaume Uni (cf. Bressoux, 1993).

Mais les écarts de niveau scolaire entre établissements sont plus faibles en France que dans ces deux pays (Grisay,1993) et l'on prendrait des risques à conclure ici d'un pays pour l'autre. Sur 17 collèges de l'académie de Dijon, suivis de 1982 à 1987, Duru-Bellat et Mingat (1993) observent deux effets de sens inverse. La progression des élèves est plus forte, toutes choses égale par ailleurs, lorsque la proportion d'élèves de milieu populaire est importante, mais plus faible lorsque "le niveau initial est bas". Sans doute faut il considérer que, lorsqu'on agrège ces deux effets, le second l'emporte sur le premier, ce qui plaiderait en faveur de l'existence d'un effet contextuel négatif préalable, donc de l'approche libérale, et donc de la possibilité que la politique ZEP ait réduit cet effet.

La nature de ces résultats, qu'il faut évidemment mettre en regard des crédits, de l'énergie et des espoirs investis dans cette politique, rend d'autant plus importante l'étude des effets non cognitifs de la scolarité en ZEP.

\section{L'EFFET DES ZEP SUR L'ÉVOLUTION DES ATTITUDES DES ÉLĖVES}

L'analyse des objectifs des ZEP et des critères de leur découpage a donné plusieurs raisons de prendre en compte le développement non cognitif dans une évaluation des ZEP. Une autre raison cependant est nécessaire, sur laquelle les avis sont contrastés. La façon dont fonctionnent les établissements scolaires a-t-elle une influence sur le comportement de leurs élèves? Rutter et al (1979) observent, sur des équivalents anglais de nos collèges, que les écoles efficaces au plan cognitif sont aussi celles où l'on constate le moins de délinquance. Brookover et al (1979) observent une relation négative entre la réussite scolaire moyenne et le fait que les élèves ont d'euxmêmes une image positive. Mortimore et al. (1988) ne constatent pas de liens entre l'efficacité scolaire d'une école, le comportement des élèves à l'école et l'attitude envers l'école. Brandsma et Knuver (1993) concluent seulement que, "les cor- 
rélations entre l'efficacité des écoles (primaires) dans les domaines cognitifs et affectifs sont petites mais jamais négatives. Ceci nous permet la conclusion prudente que l'efficacité dans les domaines affectifs et cognitifs peuvent aller de pair ".

De leur côté, A.Grisay et A. Dethier (1993) observent qu'en France, l'évolution des attitudes des élèves, est, en moyenne, faible au cours des deux premières années de collège mais qu'elle varie d'un établissement à l'autre: "L'influence de l'établissement fréquenté doit être considérée comme réelle, y compris pour l'évolution socioaffective des élèves ".

Parmi les multiples enjeux non cognitifs de l'éducation, deux ont paru, dans le cadre de cette étude, irrécusables.

Les élèves des ZEP disposent, moins que d'autres, des techniques de travail scolaire et leur rapport à l'école est particulièrement fragile. D'autre part, leur civilité - au sens donné plus haut à ce mot - est davantage à construire, comme on l'a montré. Parmi les dimensions non cognitives mesurées par la recherche sur l'efficacité des collèges, ont donc été retenues quatre variables décrivant le rapport à l'école et trois variables décrivant le rapport à autrui, en incluant les attitudes civiques dans ce dernier

Le diagnostic des élèves à l'entrée des collèges ZEP a été fait ci-dessus (III). Qu'en est-il des progrès réalisés?

Ici encore, les comparaisons de scores bruts importent moins que les comparaisons de gains corrigés. II y a souvent une corrélation entre les scores enregistrés par les élèves dans les échelles non cognitives et leurs caractéristiques individuelles : âge, appartenance à une catégorie défavorisée, nationalité et scores initiaux en français et maths. Ici encore, pour évaluer les collèges ZEP, mais tel ou tel collège aussi bien, il faut se demander si leurs élèves - ou telle catégorie de ses élèves - ont fait mieux ou moins bien que prédit par leur état individuel initial. C'est ce qui est fait dans le tableau 6 , où les gains mesurés sont les résultats de régressions des scores finals sur les scores initiaux et sur les variables que l'on vient de mentionner.

L'écart entre les gains relevés en et hors ZEP n'est pas significatif pour la moyenne des élèves, ceci pour toutes les dimensions non cognitives considérées. II est même particulièrement faible puis qu'il n'est supérieur à 0,05 écart type - au détriment des ZEP - que pour la capacité des élèves à organiser leur travail (ORG).

II arrive qu'il soit un peu plus fort, et parfois en faveur des ZEP, pour certaines catégories sociales ou scolaires - d'élèves, sans jamais atteindre, pour autant, le seuil de signification statistique. Ainsi il est probable que la motivation, la propension à la coopération, l'image de soi scolaire des élèves socialement défavorisés progressent davantage lorsqu'ils sont scolarisés en ZEP.

II est également probable que les bons élèves profitent, au moins eux, de la situation des ZEP. On compte une centaine de ces bons élèves dans les collèges ZEP de l'échantillon. Leurs capacités d'organisation, leur motivation, l'image qu'ils se font de leurs capacités scolaires, leurs attitudes civiques surtout, progressent davantage que celles des bons élèves hors ZEP, comme s'ils tiraient profit, à cet égard, soit des actions mises en place, soit de la distance plus grande qui sépare leurs compétences de celles de leurs condisciples.

Toutefois, les élèves défavorisés des collèges ZEP progressent un peu moins que prévu, et un peu moins que les élèves défavorisés scolarisés hors ZEP, pour l'organisation de leur travail (qui semble pourtant accessible à l'action de l'école et importante pour l'apprentissage)et pour la sociabilité. Les attitudes civiques et l'agressivité verbale évoluent de la même façon en ZEP et hors ZEP, à l'exception qu'on vient de signaler des bons élèves. II semble donc, à considérer ces résultats, que les collèges ZEP aient davantage réussi à améliorer le rapport au travail scolaire de leurs élèves plutôt qu'à améliorer certaines capacités que l'école, pourtant, doit dévélopper : l'organisation du travail, la civilité et la socialité.

Cette amélioration, légère, des attitudes envers la scolarisation vient-elle d'un fonctionnement particulier des collèges ZEP ? Nous rencontrerons cidessous quelques collèges ZEP où la progression à cet égard est tellement forte qu'il est sûr que leur fonctionnement y a contribué. Mais en moyenne?

Les effets de l'origine sociale de chaque élève sont tenus sous contrôle dans le tableau 6 et ne peuvent donc expliquer les écarts entre ZEP et Non ZEP. 
La question de savoir si les progressions observées pourraient venir davantage de la population scolarisée que du fonctionnement des collèges ZEP se pose néanmoins. On a pu observer, dans l'enseignement primaire spécialisé suisse (B. Pierrehumbert, 1992) que les élèves y développaient des attitudes plus favorables à l'école, au travail scolaire et on a pu montrer qu'il s'agissait non pas d'un effet de la pédagogie pratiquée dans ces classes, mais d'un plus grand conformisme des réponses des élèves "désavantagés et marginalisés par le système scolaire ", conformisme d'ailleurs interprété dans cette recherche comme un comportement rationnel d'adaptation.

On peut imaginer qu'on observe un phénomène semblable dans les ZEP françaises. D'ailleurs, Grisay (1993) observe que la présence, dans un collège, d'une forte proportion d'élèves étrangers est favorable à une évolution positive des attitudes envers la scolarisation.

Les caractéristiques du fonctionnement des collèges ZEP, quant à elles, ne paraissent pas très favorables à l'efficacité dans le domaine des attitudes envers l'école et envers autrui. Sans doute, il faut appréhender avec prudence les effets propres du fonctionnement des collèges ZEP. En effet, les modèles associant les caractéristiques des collèges à l'efficacité expliquent ici moins de la moitié de sa variance. Des variables inconnues peuvent donc avoir un impact," et être plus présentes en ZEP. Toutefois, on est obligé de constater que les variables dont on sait la corrélation avec l'efficacité en ces domaines sont plutôt moins fréquentes en ZEP que hors ZEP.

Une bonne discipline, une bonne gestion du temps en classe, une bonne couverture du programme selon les élèves, sont associées à une forte progression des attitudes envers autrui, comme si les élèves prenaient davantage modèle sur les attitudes sociales de leurs enseignants lorsque ceux-ci réussissent à les faire travailler. Ces trois caractéristiques sont moins présentes en ZEP qu'ailleurs.

La qualité de la vie au collège, une forte utilisation du CDI, l'existence d'activités de soutien sont associées à de bons progrès des attitudes envers la scolarisation.

Or la qualité de la vie est moins bonne en ZEP, l'utilisation du CDI n'y est pas plus forte. Seul le fait que les collèges ZEP pratiquent le soutien plus que les autres pourrait expliquer que, pour certains élèves, ils obtiennent des résultats un peu meilleurs sur les attitudes envers l'école.

La qualité des mesures n'est donc pas suffisante pour attribuer clairement les (très faibles) suppléments de progression observés dans les. ZEP pour certaines dimensions non cognitives, soit à un effet contextuel positif soit à la politique ZEP elle même. Elle permet seulement de dire que la question se pose.

\section{DES DIFFÉRENCES ENTRE COLLÈGES ZEP}

Le collège $X$ est un collège ZEP de l'académie de Lille. Il est un de ceux qui scolarisent la population la plus défavorisée socialement de notre échantillon. $42 \%$ de ses élèves sont étrangers et ses élèves de Gème ont en moyenne 12 ans et quart. La moitié de ses enseignant ont moins de 35 ans et le quart est non titulaire.

A bien des égards, il semble que la politique ZEP ait eu peu d'emprise sur ce collège. L'élaboration du projet d'établissement y a été fort peu collective, le Principal est peu occupé par l'activité pédagogique de son établissement, les enseignants et les élèves eux-mêmes jugent que la discipline y est très mauvaise, les relations entre élèves et enseignants sont peu chaleureuses, l'activité de soutien pédagogique est plutôt faible. II n'est donc pas étonnant qu'enseignants et parents pronostiquent une réussite très faible, et que la réputation du collège soit mauvaise, exécrable même si on en croit les enseignants.

Si l'on classait, cependant, les 100 collèges de l'échantillon selon les progrès accomplis par leurs élèves en français et en maths, leur niveau initial étant tenu sous contrôle, $X$ se classerait à peu près au $15^{\mathrm{e}}$ rang $(+1,1 \sigma)$. L'efficacité est plus forte encore si l'on s'intéresse non plus à l'efficacité moyenne, mais à l'objectif d'éviter que les écarts ne se creusent entre élèves initialement forts et faibles $(1,3 \sigma)$. Ces résultats sont d'autant plus remarquables que les progressions sont ici corrigées du seul niveau initial de chaque élève, ne tiennent donc pas sous contrôle les handicaps supplémentaires qui peuvent peser sur ce collège. Les attitudes des élèves envers l'école et le travail scolaire évoluent, elles aussi, à niveau initial égal, significativement mieux que dans la moyenne des autres collèges $(+0,6 \sigma)$ : c'est particulièrement vrai de l'image de soi scolaire $(+1,5 \sigma)$, mais aussi des autres attitudes prises en compte: 
motivation, stratégies positives, sentiment de maîtrise, mobilisation. Seule fait exception la capacité à organiser son travail $(-0,6 \sigma)$. Les attitudes envers la socialisation évoluent, elles, comme dans la moyenne des collèges, avec cependant - une exception positive (les attitudes civiques évoluent mieux qu'ailleurs) et une négative (la propension à la coopération évolue moins bien qu'ailleurs).

Ces résultats satisfaisants peuvent, semble-t-il, être rapprochés de deux caractéristiques de ce collège. D'abord un environnement mobilisé. Les déclarations des élèves situent la façon dont leurs parents les aident, suivent leur travail, valorisent leurs études, bien au dessus de la moyenne. "I semble, selon le Principal, que ce suivi soit aussi l'œuvre d'une "école des devoirs", prise en charge par une association en dehors du temps scolaire. Si pessimisme il y a de la part des parents, c'est donc un pessimisme actif. Deuxièmement, les enseignants ne rejettent pas le collège ni ses élèves. Ils pratiquent un enseignement beaucoup plus structuré que dans la moyenne des collèges, plutôt frontal, relativement peu a centré sur l'élève ». lls n'ont cependant pas renoncé à innover (les réponses des enseignants situent le collège dans la moyenne pour le recours aux PAE, aux actions financées par le Fonds d'Aide à l'Innovation, et pour l'introduction récente d'innovations dans le cours). Ils enseignent dans des classes qui, en 6ème, sont de petite taille. Les élèves déclarent avoir étudié en classe une grande partie de l'épreuve de maths, ce qui tend à montrer non seulement que le programme est " fait " dans une large mesure, mais aussi qu'il est " fait " de telle sorte que les élève se souviennent qu'il l'a été. Et de fait, relativement peu de temps est perdu pendant les cours $(-0,4 \sigma)$, nettement moins qu'on ne pourrait s'y attendre. Les élèves se sentent pris en considération (on leur fait plutôt confiance, on accepte de les écouter, les enseignants s'intéressent à ce qu'ils font : $1,5 \sigma$ ) ; ils portent un jugement plutôt positif sur leurs enseignants $(+0,4 \sigma)$, bien que ceux ci ne les appellent par leur prénom, ne les tutoient, moins souvent qu'ailleurs.

En retour, si l'on peut dire, les enseignants de ce collège sont plutôt contents de la vie qu'ils y mènent $(+1 \sigma)$, comme d'ailleurs le chef d'établissement.

Le collège $X$ est significatif à plusieurs titres :

- il montre qu'il existe des collèges ZEP efficaces. De fait, à partir du nombre de collèges
ZEP qui se situent à plus d'un écart type de la performance moyenne des collèges ZEP, on peut estimer quie 4 à 6 des 31 collèges ZEP de notre échantillon, sont parmi les 34 collèges les plus efficaces pour le français et les maths, parmi les 12 collèges les plus efficaces pour les attitudes envers la socialisation, parmi les 10 collèges les plus efficaces pour les attitudes envers la scolarisation (6).

- il montre que l'efficacité peut naître de combinaisons inattendues mais pas forcément complexes: ici un cercle vertueux semble s'être engagé à partir de deux éléments seulement, la qualité du suivi scolaire et la pratique des enseignants. Une conséquence en est, qu'il serait présomptueux de juger de l'efficacité d'un collège ZEP d'après le simple examen de ses activités, fut-il aussi détaillé et précis qu'ici. A. Grisay (1993) fait remarquer que ce collège peu résigné est classé par l'analyse hiérarchique dans une catégorie que ses traits distinctifs l'ont précisément conduite à appeler " résignée ".

C'est pourquoi l'on s'est attardé sur son cas avant de relier d'une façon générale le fonctionnement des collèges ZEP et leurs résultats.

Sur les 100 collèges de notre échantillon, une analyse hiérarchique a permis de distinguer huit profils de collèges (Grisay, 1993), d'après les modalités de leur, fonctionnement. Chaque profil contient au moins un collège ZEP, ce qui illustre la diversité des situations que l'on peut rencontrer en ZEP. Nous analyserons seulement les collèges ZEP de trois profils $(1,2,8)$. Les collèges ZEP des autres profils ont été jugés trop hétérogènes (3), pas assez "défavorisés " socialement (4), ou encore, trop peu nombreux (un par profil). Le profil des 18 collèges ZEP restant est présenté dans le tableau 7, lequel présente les performances des trois groupes et les variables élémentaires qui les distinguent le plus.

Les collèges ZEP du groupe 2 sont ceux où la plus forte probabilité est que la politique ZEP n'a rien changé, si elle n'a pas aggravé les choses. Les élèves y progressent moins qu'ils ne le devraient, au vu de leurs compétences initiales, dans les trois dimensions. Le climat (discipline, gestion du temps, qualité de la vie, ...) y est mauvais. On y trouve les actions habituellement prônées aux ZEP (soutien scolaire, connaissance des parents d'élèves, innovations pédagogiques, participation des enseignants aux décisions) plutôt moins fréquemment que dans le collège moyen 
Tableau 7. - Trois profils de collèges ZEP

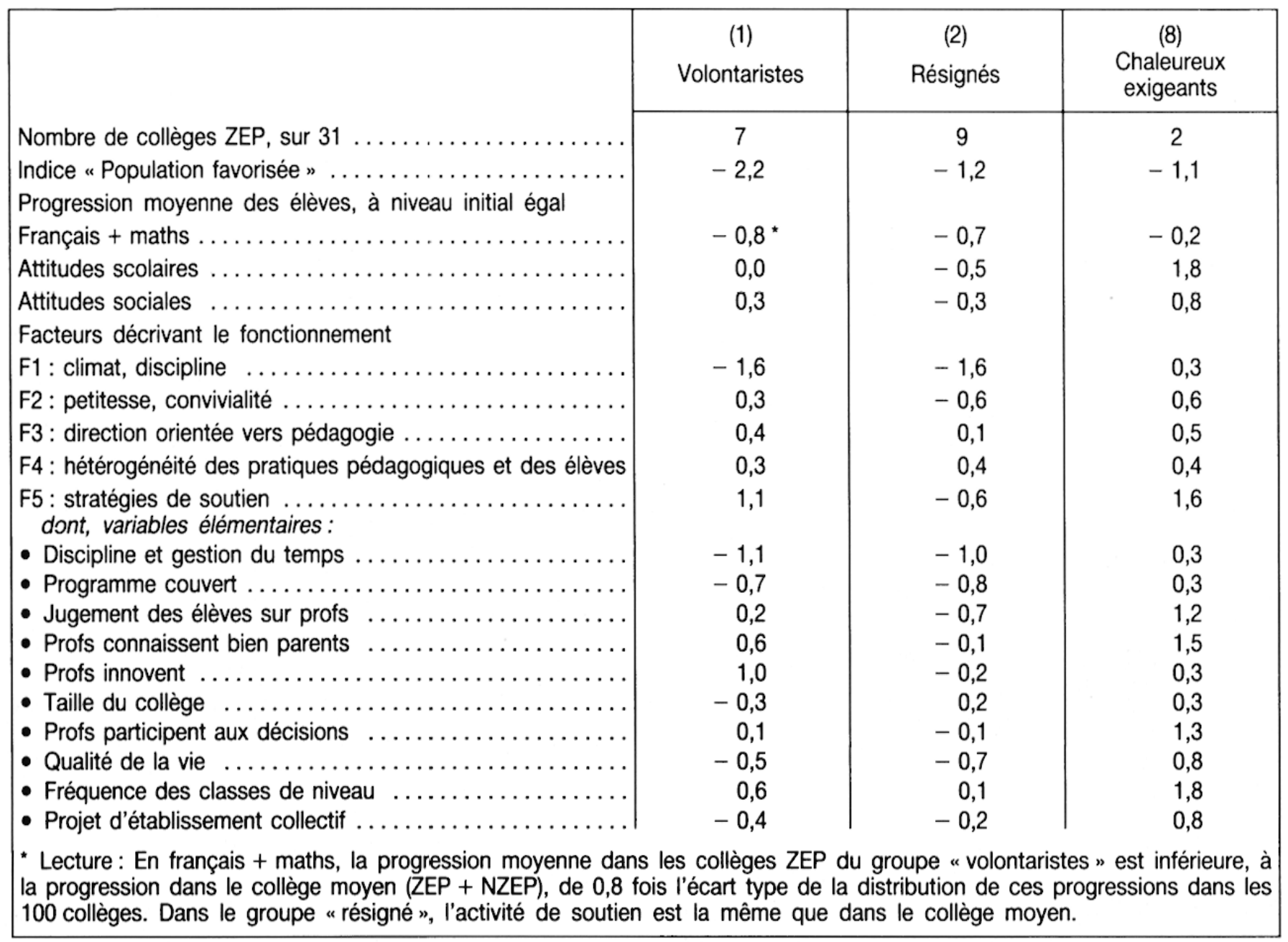

(ZEP ou NZEP). Le jugement des élèves sur les enseignants est sévère.

Par contraste, les deux autres groupes dessinent deux objectifs possibles à la politique ZEP. Le groupe 1 réussit à ne pas faire plus mal que le 2, voire un peu mieux, alors que la population y est beaucoup plus défavorisée et que donc on peut imaginer un effet contextuel négatif beaucoup plus fort. Ces collèges sont plus petits que les précédents, pratiquent davantage le soutien scolaire, leurs enseignants innovent davantage, connaissent mieux les parents de leurs élèves, la répartition des élèves dans les classes tient davantage compte du niveau scolaire des élèves. Les élèves jugent de façon plus positive leurs enseignants.

Le groupe 8 comprend seulement deux collèges ZEP. Ils réussissent à obtenir des résultats nettement meilleurs que ceux du groupe 2 en scolari- sant une population aussi défavorisée que lui. Le climat, la discipline, la convivialité y sont de loin meilleures, un peu meilleures même que dans la moyenne des collèges. Les professeurs participent aux décisions, comme à l'élaboration du projet d'établissement. Ils connaissent bien les parents, développent une forte activité de soutien scolaire. Ces collèges comprennent des classes de niveau . Le jugement des élèves sur leurs enseignants est parmi les plus positifs qui soient, et la qualité de la vie est bonne. Ceci ne s'accompagne pas d'un relâchement des exigences scolaires: le programme est couvert un peu plus que dans la moyenne des collèges ; le temps passé à travailler en classe, la discipline y sont aussi plus grands.

Si nous décidons d'appeler échec de la politique ZEP le fait qu'elle ait, clairement, échoué à supprimer l'écart entre les collèges défavorisés et les autres, ou encore le fait qu'elle ait tout aussi clairement, réussi moins bien que ses promoteurs 
ne l'espéraient, une des questions principales posées par cet échec est celle de savoir pourquoi, quand onze collèges ZEP au moins de notre échantillon pouvaient atteindre le profil "chaleureux exigeant ", deux seulement l'on fait.

\section{CONCLUSION}

Dans les collèges ZEP étudiés - qui scolarisent, rappelons-le, une population un peu plus difficile que l'ensemble des collèges ZEP de la France métropolitaine - les effets de la politique ZEP sont faibles. Une interprétation prudente des chiffres conclurait seulement que les effets obtenus sont très faibles au regard des espoirs qu'on $y$ avait mis.

Cette politique n'a pas réussi à faire en sorte que les élèves défavorisés ou faibles aient, en ZEP, davantage ou autant de chances qu'ailleurs de progresser en maths et en français - soit qu'elle ait été impuissante à combler un sur-handicap territorial qui précédait l'instauration de cette politique, soit qu'elle ait pu en créer un léger.

Les effets de la politique ZEP sur les attitudes scolaires des élèves sont tout aussi faibles, mais, pour certaines d'entre elles au moins (motivation, capacité à ne pas se décourager) la tendance semble positive, encore que statistiquement non significative. Si tel est le cas, ces gains peuvent avoir de bons effets à moyen terme, en contribuant à éviter que ces élèves ne rejettent l'école, et, plus généralement, l'idée même d'apprentissage.

Les attitudes des élèves envers autrui évoluent, en moyenne, dans les ZEP comme elles évoluent ailleurs.

Enfin, les effets de la politique ZEP semblent différer, selon les catégories d'élèves concernés et selon les modalités de fonctionnement du collège.

Les élèves "défavorisés" subissent dans les ZEP un handicap plus grand qu'ailleurs quant à leur progression en français et en maths. Leurs attitudes envers l'école y évoluent un peu plus favorablement qu'ailleurs, sans qu'il soit aisé de distinguer les parts qui en reviennent aux actions conduites ou à un plus grand conformisme vis-àvis de l'institution scolaire.
II est apparu que les bons élèves des ZEP étaient, paradoxalement, les principaux bénéficiaires de cette politique, avec les conséquences heureuses que cela peut avoir pour la fréquentation de ces collèges et la vie de leur quartier.

II est apparu aussi que la politique ZEP n'avait pas homogénéisé la manière dont les collèges font face à des populations défavorisées. En effet, les effets faibles, souvent faiblement négatifs, parfois faiblement positifs, que l'on peut attribuer à la politique ZEP semblent moins le résultat d'une loi d'airain, selon laquelle tous les efforts en vue d'une plus grande égalité devant l'enseignement sont voués à l'échec, que de l'existence de plusieurs modèles de collèges, qui obtiennent des effets différents et qui se compensent.

Au total, si l'on suppose qu'il n'existe pas d'effet contextuel négatif dans les ZEP, il faut conclure à l'inutilité, peut être à la nocivité, de la politique ZEP dans les collèges. A titre d'explication, on pourrait avancer que les effets négatifs de "l'étiquetage " ZEP sur les attentes et les exigences des acteurs l'ont emporté sur les effets positifs de projets mobilisateurs.

Si l'on suppose qu'il existait, avant la politique ZEP, un effet contextuel négatif, le bilan est plus incertain, probablement moins négatif. La politique ZEP n'a pas réussi à supprimer en France cet effet contextuel négatif, mais elle a parfois réussi à le limiter, à l'inverser dans certains établissements.

On se trouve alors dans le cas de constater l'efficacité pour le moins limitée d'une politique en même temps qu'on donne un argument de plus en faveur de sa nécessité du point de vue de la justice. II s'avère en effet que les conditions de scolarisation (discipline, convivialité, taille du collège, etc.) ne sont pas identiques dans les collèges fréquentés plutôt par des élèves favorisés et ou défavorisés. Une politique de discrimination positive apparaît dès lors justifiée, non seulement pour compenser les conditions externes qui viennent gêner l'usage que les élèves pourraient faire de ressources identiques, mais aussi parce qu'en réalité les ressources ne sont pas identiques, si l'on veut bien englober sous ce terme l'ensemble de la qualité de l'environnement scolaire.

Cette étude permet peut être d'avancer quelques propositions pour une discrimination positive plus efficace.

L'un des profils de collège mis en évidence semble indiquer que, dans nombre de collèges 
ZEP, prévaut la situation même que cette politique devait supprimer. Ce profil incite à proposer que les résultats obtenus, comme les actions réellement menées, jouent un plus grand rôle dans le maintien des établissements au bénéfice de cette politique. II suggère aussi que l'on s'efforce de comprendre pourquoi certains établissements ont pu évoluer vers un fonctionnement qui semble adapté à leur public quand d'autres n'ont pu le faire.

Par ailleurs, cette étude suggère que l'articulation entre une politique de la ville et une politique d'égalisation des chances éducatives doit être, sans doute, pensée avec plus de précision qu'elle ne l'est actuellement, où l'on postule que ce qui est bon pour l'une est forcément bon pour l'autre.

D'un côté, ces deux politiques sont complémentaires. Certains fonctionnements d'établissement peuvent servir à la fois l'une et l'autre. C'est un résultat qui n'était pas évident pour tous. Par exemple, on peut, à partir de Grisay (1993), émettre l'hypothèse que la mobilisation des enseignants au profit de l'enseignement, en particulier des élèves défavorisés, s'accompagne pour ces élèves d'une amélioration de la relation à autrui. On peut aussi en tirer l'idée que diminuer la délinquance et la violence dans un établissement scolaire est sûrement favorable à la progression des élèves en français et en mathématiques.

D'un autre côté, cette étude (en particulier les collèges du profil 8) suggère qu'il est peut être plus facile, pour un collège ZEP, de faire mieux que les autres pour la progression " non cognitive" des élèves que pour leur progression " cognitive». Dune façon générale, la politique
ZEP semble avoir mieux réussi en tant que contribution de l'Éducation nationale à la Politique de la ville (effet sur les bons élèves; sur les attitudes des élèves défavorisés ou en difficulté envers la scolarisation et la socialisation) qu'en tant que politique de lutte contre l'échec scolaire. Est-ce, tout simplement, parce qu'elle a été la première plus que la seconde ? Est-ce parce qu'il est (un peu) moins difficile de réussir dans le premier domaine que dans le second?

Peut-être y aurait-il intérêt à distinguer davantage ces deux politiques. L'unité pertinente pour une politique de lutte contre l'échec scolaire n'est probablement ni la zone, ni l'établissement, mais l'élève lui-même. On pourrait imaginer que l'on attribue aux établissements une dotation horaire tenant compte du nombre d'élèves à fort pronostic d'échec. La contribution du ministère de l'Éducation nationale à la politique de la ville, doit, elle, s'appuyer sur tous les établissements d'une zone. Elle peut poursuivre des objectifs partiellement différents de la première: par exemple, viser l'excellence pour certains élèves, améliorer les méthodes de travail des élèves moyens, éviter la désocialisation des plus faibles. II est possible que certains établissements soient capables et désireux d'assurer cette contribution à la vie de leurs quartiers par une politique visant la réussite scolaire de tous leurs élèves, et il va de soi qu'ils devraient y être encouragés. Mais il n'est pas sûr que ce soit le cas de tous les collèges qui scolarisent une forte proportion d'élèves en difficulté.

Denis Meuret

Ministère de l'Éducation nationale Direction de l'Évaluation et de la Prospective 1992-1993 IUFM de Bretagne 1993-1994

\section{NOTES}

- Ce travail a été réalisé à partir des données collectées pour l'étude sur les facteurs d'efficacité des collèges (1990-1992), menée dans le cadre d'une convention de recherche du Ministère de l'Education nationale (Direction de l'évaluation et de la Prospective), par A. Grisay, A. Dethier et G. Hindryck, (Université de Liège, service de Pédagogie Expérimentale), D. Meuret, Ph. Abi-Saab et D. Trancart (MEN, DEP 11, Paris). II n'engage cependant pas la responsabilité de la Direction de l'Evaluation et de la Prospective. II a été commencé alors que j'appartenais à la DEP qu'il ne saurait néanmoins engager. Je suis redevable à A. Grisay des traitements statistiques et informatiques, ainsi que d'une lecture vigilante de sa première version. On trouvera dans Grisay (1993) une présentation générale de l'étude sur les facteurs d'efficacité des collèges et, en particulier, des notions utili- sées dans ce travail pour apprénender les attitudes des élèves.

(1) Il est possible d'ailleurs que l'éparpillement des moyens sur un trop grand nombre d'écoles et de zones, que les textes ministériels stigmatisent dès le second d'entre eux (circulaire du 28/12/81) et constamment ensuite, ait, en partie, son origine dans la conscience de ce risque.

(2) La DEP confirme cet accroissement de la précarité, plutót sous la forme d'un accroissement de l'écart entre les ZEP et le reste du système, sur une série de paramètres décrivant la population prise en charge (retards scolaires, nationalité...) (Liensol, MENC-DEP, 1992), mais non la première partie de l'appréciation. 
(3) L'évaluation (sur 58 élèves-cible et 65 élèves d'un groupe témoin) d'un programme intensif de soutien distribué voici 30 ans aux écoliers socialement défavorisés de maternelle de Ypsilanti (Michigan), vient de conclure à des effets positifs et importants de ce programme sur la vie et les revenus des anciens élèves, observés à l'âge de 27 ans. Selon le rapport, 15 investi dans le programme a rapporté $7 \$$ à l'État, en diminution de prestations sociales, de frais de justice et de police, et en impóts supplémentaires perçus sur des revenus supérieurs. Ces effets positifs à long terme ont été précédés d'effets positifs sur le langage et ml'intelligence a des enfants jusqu'à l'áge de 7 ans, effets qui se sont ensuite estompés, et d'effets positifs sur certaines compétences scolaires, appréhendées à 14 et 19 ans. Cette évaluation semble donc plaider pour l'existence d'une relation non nulle, mais complexe, entre effets à court et long terme. (Cohen D.L., 1993).

(4) Cet artefact, décrit par Cook et Campbell (1979) est lié à l'inévitable erreur de mesure dans les tests initial et final, ainsi qu'à la corrélation existant entre score initial et appartenance à l'un ou à l'autre des groupes que l'on compare (ici : ZEP ot hors ZEP).
(5) Rappelons que *milieu social défavoriséa est défini dans cette étude de façon stricte ce qui limite le risque que ce résultat provienne de ce que les personnes "défavorisées" de ZEP le seraient davantage que les autres. (Cf. note $1 \mathrm{du}$ tableau 1).

(6) Cette estimation suppose que la distribution des performances des 100 colléges est mnormalea, elle est donc très approximative. Le raisonnement est, sur un exemple : la performance cognitive du collège ZEP moyen est à - 0,59 o de la performance du collège moyen. L'écart type de la distribution des performances des collèges ZEP est de 0,96 o (la dispersion de leurs performances est donc presque aussi grande que celle de l'ensemble des collèges...). En conséquence, on peut estimer que $16 \%$ des 31 collèges ZEP, soit environ 5 collèges sont situés à $-0,59+0,96=+0,37$ écart type de la performance du collège moyen. Cela revient à dire que ces cinq collèges appartiennent aux 34 collèges plus performants de l'échantillon d'ensemble. Les valeurs equivalentes pour SCO sont $m=0,2 \sigma=1,1$, pour SOC $m$ $=0,2 \sigma=0,96$.

\section{BIBLIOGRAPHIE}

BLOOM B.-S. (1984). - Le défi des deux sigmas : trouver des méthodes d'enseignement collectives aussi efficaces qu'un précepteur, in CRAHAY M. et LAFONTAINE D., éd., L'art et la science de l'enselgnement, Éditions Labor, Liège.

BRESSOUX P. (1993). - Les performances des écoles et des classes, MEN DEP, Dossiers Éducation ot Formations, $n^{\circ} 30$.

BRIZARD A., RADICA K. (1992). - Les résultats en termes d'acquis d'élèves: les évaluations CE2- ${ }^{\circ}$ de 1989 et 1991. Comparaison des résultats en ZEP ot hors ZEP, in MENC-DEP, p. 30.

BROOKOVER et al (1979). - School social systems and student achievement, Schools can make a difference, New-York, Bergin Publishers Book.

CHAMBON A. et PROUX M. ( 1988). - Zones d'Éducation Prioritaires : un changement social en éducation ? in Revue Française de Pédagogie, $n^{\circ} 83$.

CHAUVEAU G. et DURO-COURDESSES L. (1989.). Écoles et quartiers : des dynamiques éducatives 10cales, Paris, I'Harmattan/INRP.

CHERKAOUI M. (1979). - Les paradoxes de la réussite scolaire, PUF.

CHRETIENNOT C. (1985). - Les « Zones d'Éducation Prioritaires" et les “Écoles socialement prioritaires » en Grande-Bretagne : éléments d'information, in CRESAS.

COHEN D.-L. (1993). - Perry presschool graduates show dramatic new social gains at 27 , in Education week, vol. 12, $n^{\circ} 30,21$ avril.

COOK T.-D. et CAMPBELL D.-T. (1979). - Quasi-expérimentation, design and analysis issues for field settings, Chicago, Rand Mac Nally.

DONZELOT J., ESTEBE Ph. (1994). - L'Etat animateur, Essai sur la politique de la ville, Paris, Éditions Esprit.

DURU-BELLAT M., JAROUSSE J.-P. et MINGAT A. (1992). - Les inégalités sociales de carrière du cours préparatoire au second cycle du secondaire, cahiers de l'IREDU, $n^{\circ} 51$, janvier.
DURU-BELLAT M. et MINGAT A. (1993). - Pour une approche analytique du fonctionnement du systeme educatif, Paris PUF.

GLASMAN D. et AL (1991). - Le soutien scolaire hors école, in Revue Française de Pédagogie, $n^{\circ} 95$, avril-juin, Paris.

GRISAY A. et DETHIER A. (1993). - Les deux premières années du collège : le progrès des connaissances contraste avec l'évolution moins positive des méthodes de travail et des " Savoir-être ", Éducation et Formations, $n^{\circ} 34$.

GRISAY A. (1993). - Le fonctionnement des collèges et ses effets sur les élèves de $6^{\theta}$ et $5^{\theta}$, MEN-DEP, Dossiers Education et Formations, $n^{\circ} 32$.

HENRIOT van ZANTEN (1988). - Les ressources du local, innovation pédagogique et changement social dans les ZEP, in Revue Française de Pédagogie, $n^{\circ} 83$, pp. 23-30.

HEUCHENNE C. (1993). - Effets de l'erreur de mesure des covariables sur l'évaluation de l'efficacité d'un traitement, in Mathématiques, Informatique ot Sciences humaines, $\left.X X X\right|^{\ominus}$ année, $n^{\circ} 122$, pp. 5-18.

ISAMBERT JAMATI V. (1973). - Les " handicaps socioculturels " et leurs remèdes pédagogiques, in P'Orientation Scolaire ef Professionnelle, $n^{\circ} 4$.

ISAMBERT JAMATI V. (1989). - Les choix éducatifs dans les zones prioritaires, in Revue française de sociologie, $n^{\circ} 31$, pp. 75-100.

KNUVER A. et BRANDSMA H.-P. (1993). - Cognitive and affective outcomes in school effectiveness research, University of Twente, the Netherlands, School effectiveness and school improvement, vol. $4, n^{\circ} 3$.

LIENSOL B. et CEUVRARD F. (1992). - Le fonctionnement des ZEP et les activités pédagogiques des établissements, in MENC-DEP.

MINGAT A. (1983). - Evaluation analytique d'une action Zone d'Éducation Prioritaire au cours préparatoire, cahiers de l'IREDU, $n^{\circ} 37$, septembre. 
MORTIMORE P. et al. (1988). - School matters: the junior years, Somerset, open Books.

MORTIMORE J. et BLACKSTONE T. (1982). - Divadvantage and Education, studies in deprivation and disadvantage 4 London, Heinemann Educational Books, London.

PIERREHUMBERT B. (1992). - L'échec à l'école : Echec de l'école ?, Neuchâtel, Delachaux et Niestlé, chapitre V.

PLAISANCE E. (1988). - La Politique des Zones d'Éducation Prioritaires et sa réalisation, in handicaps et inadaptations, les cahiers du CTNERHI, $n^{\circ} 43$, juillet-septembre, pp. : 1-9.

ROSS K. (1983). - Social area indicators of educational need, ACER research monograph $n^{\circ} 20$, Hawthorn (Victoria), Australie.

RUTTER M. et al ( 1979). - Fifteen thousand hours, secondary schools and their effects on children, London, Open books.

SLAVIN R.-E. ( ). - Achievement effects of ability grouping in secondary schools: a best evidence synthesis, in Review of educational research, pp. 471-179, Fall 199 , vol. $60, n^{\circ} 30$.

SCHEERENS J. (1992). - Effective schooling : research, theory and practice, London, Cassel.
TRANCART D. (1992). - Les collèges on ZEP et les autres: typologie des collèges et classement on ZEP, in MENC-DEP.

TRANCART D. (1992). - Disparités entre collèges publics in Education et Formations, $n^{\circ} 31$.

Académie de Créteil (1986). - L'évaluation régulatrice en ZEP, CRDP 93.

CRESAS (1985). - Depuis 1981, l'école pour tous ? Zones d'Education Prioritaires, collection CRESAS $n^{\circ} 4$.

Inspection générale de l'Administration de l'Éducation nationale (1990). - Rapport général 1988-1989, Paris, la Documentation française, juillet.

IGEN (1986). - Rapport relatif aux Zones Prioritaires, MEN.

IGEN-IGEAN (1992). - Rapport à M. le Ministre d'État relatif aux zones d'éducation prioritaire, mission des Inspections générales, MENC, juin.

MENC-DEP (1992). - l'Évaluation des Zones d'Éducation Prioritaires : description, typologie, fonctionnement, résultats, Dossiers Éducation et Formations, $n^{\circ} 14$.

PLOWDEN (1967). - Report: Children and their primary schools, report of the central advisory council for Education in England, HMSO, London.

\section{Annexe 1 - L'échantillon}

Cette étude porte sur trente et un collèges publics, classés en ZEP en 1990-91, appartenant aux académies d'Amiens (3), de Dijon (5), de Grenoble (4), de Lille (13), de Montpellier (4), de Toulouse (2). Sur ces trente et un, trois sont en ZEP depuis seulement 1990. Ces collèges sont comparés à 69 collèges hors ZEP, au sein d'un échantillon de 100 collèges présenté dans Grisay (1993).
Les caractéristiques de l'échantillon de 31 collèges, par rapport à l'ensemble des collèges ZEP, sont les suivantes. Certaines sont données pour 1989-90 parce qu'elles n'étaient disponibles aux trois niveaux que pour cette année là.

Les 31 collèges ZEP de l'échantillon sont en moyenne dans une situation plus difficile que l'ensemble des collèges ZEP. En répartissant les

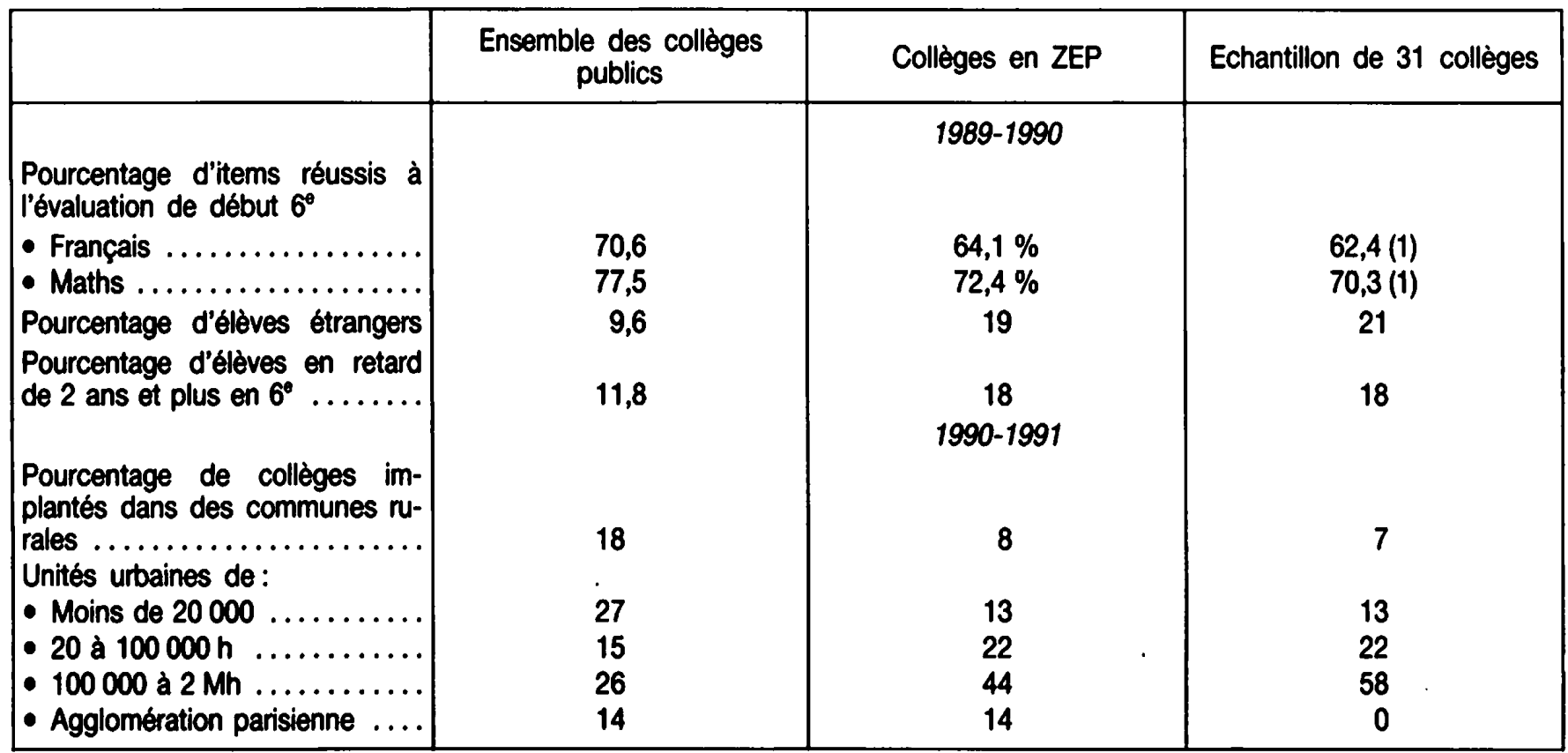

(1) Cette étude a utilisé l'évaluation de $6^{\circ}$ de 1990-1991, plus * difficile * que celle de 1989-1990. De là les différences avec les résultats présentés dans les tableaux 1 et 3. 
élèves en trois catégories selon leur origine sociale on trouve, pour l'année 92-93, que les 31 collèges scolarisent $68 \%$ de défavorisés (contre $63 \%$ pour l'ensemble des ZEP) $18 \%$ ' de " moyens " (contre $22 \%$ ) et $14 \%$ de " favorisés " (contre $15 \%$ ). (Données communiquées par MEN/ DEP). La catégorie défavorisée est ici définie de façon plus large que dans le corps de l'étude.
Cela est confirmé par la comparaison entre ces collèges et ceux qu'une analyse hiérarchique (Trancart, 1992) a permis de classer comme "en difficulté " ou "sensibles": Les deux tiers des collèges ZEP étudiés ici sont des collèges repérés comme "sensibles" contre moins de la moitié pour l'ensemble des collèges ZEP.

\section{Annexe 2 - Lisrel}

Le modèle statistique de structure de covariances, dont LISREL est le logiciel le plus connu, combine trois modèles :

- une analyse factorielle de variables observées explicatives en des variables latentes exogènes spécifiées ;

- une analyse factorielle de variables observées critères en des variables latentes endogènes spécifiées ;

- une régression linéaire des variables endogènes sur certaines variables exogènes (et même d'autres endogènes) exprimant les relations causales fondamentales.

Une de ses caractéristiques intéressantes pour notre problème est la possibilité qu'il offre de définir a priori des facteurs latents, mesurés par des indicateurs effectivement observés. Le modèle permet de délester ces facteurs latents de l'erreur de mesure que comportent bien évidemment les variables observées. Ce sont ces variables latentes "épurées" que le modèle utilise pour estimer les paramètres de l'équation de régression, ce qui a pour effet d'écarter les risques d'artefact évoqués dans le texte.

Le modèle mis en œuvre (figure 1) comporte 16 variables observées, soit:

- 4 scores de post-test (variable critère)

- 8 scores de prétest (variables de contrôle)

- 3 variables descriptives de l'élève :

- «CSP défavorisée (1) ou non (0)« (variables de contrôle)

- "Nationalité française (0) ou non (1)" (variables de contrôle)

- "âge" (variables de contrôle)

- la variable ZEP-NZEP (variable de traitement)

On a considéré que les variables CSP, nationalité, âge et ZEP/NZEP sont relevées sans erreur de mesure (ou, plutôt, on a considéré comme négligeable cette erreur). On postule, par ailleurs, l'existence de deux facteurs latents PRE (compétence initiale des élèves en français et en mathématiques) et POST (compétence des élèves deux ans plus tard), qui saturent les autres variables observées (respectivement, les 8 scores de pré et les 4 scores de post-test).

La solution obtenue peut être considérée comme satisfaisante (indice d'ajustement: 99 ; différence maximum en valeur absolue entre covariances observées et covariances estimées par le modèle : 0,039 ).

Sous contrôle des caractéristiques de l'élève à l'entrée en sixième (CSP, nationalité, âge et compétences initiales), l'effet net de l'appartenance à un collège ZEP est bien négatif, mais extrêmement léger ( $\beta$ : - 0042).

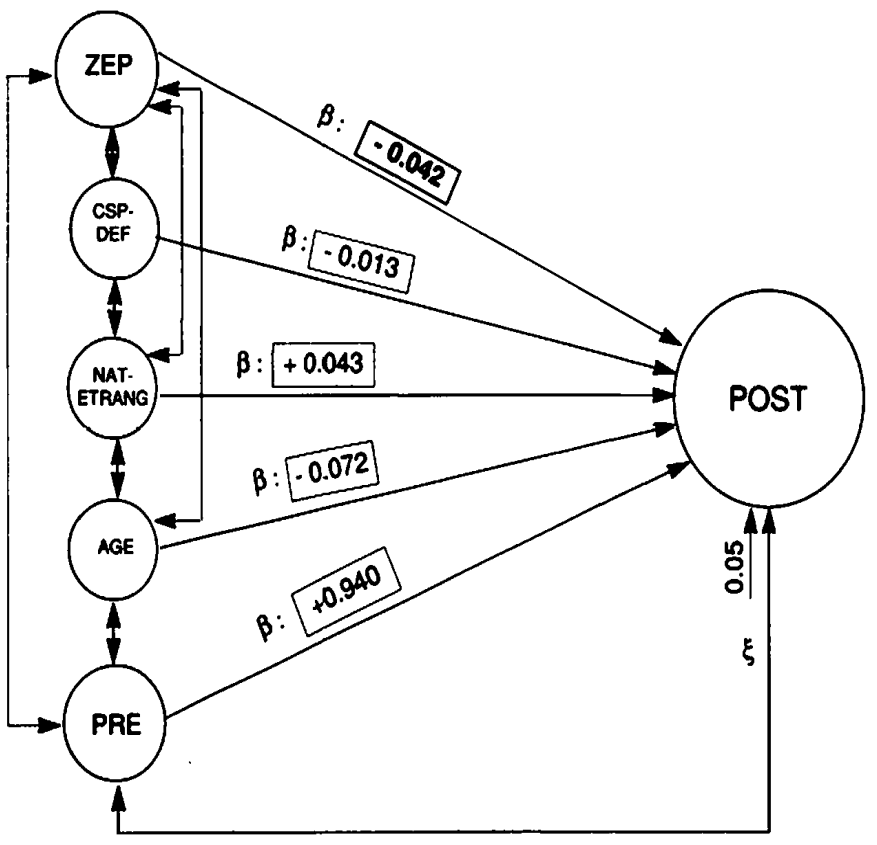

Figure 1. - Estimation par anahse LISREL de l'impact de variable "appartenance à un college ZEP " sur les compétences do l'élove deux ans apres son entré on sixieme 